\title{
The boundary degeneracy theory of a strongly degenerate parabolic equation
}

Huashui Zhan*

\section{${ }^{*}$ Correspondence:}

2012111007@xmut.edu.cn

School of Applied Mathematics,

Xiamen University of Technology,

Xiamen, 361024, China

\begin{abstract}
A kind of strongly degenerate parabolic equations,

$$
\frac{\partial u}{\partial t}=\frac{\partial}{\partial x_{i}}\left(a^{i j}(u, x, t) \frac{\partial u}{\partial x_{j}}\right)+\frac{\partial b_{i}(u, x, t)}{\partial x_{i}}, \quad(x, t) \in \Omega \times(0, T),
$$

is considered. The paper first shows that the solution of the equation may be free from the limitation of the boundary value condition. The key is to determine the portion of the boundary on which we can impose the homogeneous boundary value. By introducing a new kind of entropy solution matching the partial boundary condition, the existence of the solution is obtained by the parabolic regularization method, and the stability of the solutions is obtained by Kruzkov's bi-variables method combined with an elegant partition technique.
\end{abstract}

MSC: $35 \mathrm{~L} 65 ; 35 \mathrm{~K} 65 ; 35 \mathrm{~K} 55$

Keywords: boundary degeneracy theory; degenerate parabolic equation; partial boundary condition; entropy solution; partition technique

\section{Introduction}

The author studies the boundary condition of a kind of degenerate parabolic equations

$$
\frac{\partial u}{\partial t}=\frac{\partial}{\partial x_{i}}\left(a^{i j}(u, x, t) \frac{\partial u}{\partial x_{j}}\right)+\frac{\partial b_{i}(u, x, t)}{\partial x_{i}}, \quad \text { in } Q_{T}=\Omega \times(0, T),
$$

where $\Omega \subset \mathbb{R}^{N}$ is an open bounded smooth domain, $\left(a^{i j}\right)$ is a symmetric matrix with nonnegative characteristic values, i.e. for any $\xi \in \mathbb{R}^{N}$,

$$
a^{i j}=a^{j i}, \quad a^{i j} \xi_{i} \xi_{j} \geq 0,
$$

and we specially assume that

$$
a^{i j}(0, x, t)=0, \quad i, j,=1,2, \ldots, N
$$

Equation (1.1) arises in many applications, e.g. the porous medium equation

$$
\frac{\partial u}{\partial t}=\Delta u^{m}
$$

(c) 2016 Zhan. This article is distributed under the terms of the Creative Commons Attribution 4.0 International License (http://creativecommons.org/licenses/by/4.0/), which permits unrestricted use, distribution, and reproduction in any medium, provided you give appropriate credit to the original author(s) and the source, provide a link to the Creative Commons license, and indicate if changes were made. 
the equation in the boundary layer theory,

$$
w^{2} w_{\eta \eta}-w_{\tau}-\eta U w_{\xi}+A w_{\eta}+B w=0
$$

where $A, B$ are two known functions derived from the Prandtl system, one may refer to [1] for details. Clearly, equation (1.1) is of a hyperbolic-parabolic mixed type and might have a discontinuous solution. For the Cauchy problem of equation (1.1), whether it is weakly degenerate or strongly degenerate is a question that has been deeply investigated. For the initial-boundary value problem of equation (1.1), we know that the initial value condition is always necessary,

$$
u(x, 0)=u_{0}(x), \quad x \in \Omega .
$$

But the question is whether can we impose the Dirichlet homogeneous boundary condition

$$
u(x, t)=0, \quad(x, t) \in \partial \Omega \times(0, T)=\Sigma \times(0, T)
$$

as usual. Is (1.6) overdetermined? Let us observe a special example. Consider

$$
\frac{\partial u}{\partial t}=\frac{\partial}{\partial x_{i}}\left(d^{\alpha}(x, t) a(u) \frac{\partial u}{\partial x_{i}}\right), \quad \text { in } Q_{T}
$$

where $d(x)=\operatorname{dist}(x, \partial \Omega)$ is the distance function from the boundary, $\alpha>0$ is a constant. Suppose equation (1.1a) has a classical solution. For any given positive integer $m$, let $g_{m}(s)$ be an odd function. When $s \geq 0$, it is defined as

$$
g_{m}(s)= \begin{cases}1, & s>\frac{1}{m}, \\ m^{2} s^{2} e^{1-m^{2} s^{2}}, & s \leq \frac{1}{m} .\end{cases}
$$

If $u$ and $v$ are two classical solutions of equation (1.1a) with the initial values $u_{0}, v_{0}$, respectively, denoting $A^{\prime}(s)=a(s)$, then we have

$$
\begin{aligned}
\int_{\Omega} g_{m} & (A(u)-A(v)) \frac{\partial}{\partial t}(u-v) d x \\
= & -\int_{\Omega} d^{\alpha}(x, t)\left[a(u) \frac{\partial u}{\partial x_{i}}-a(v) \frac{\partial v}{\partial x_{i}}\right] g_{m}^{2}(A(u)-A(v)) d x \\
& -\int_{\partial \Omega} d^{\alpha}(x, t)\left[a(u) \frac{\partial u}{\partial x_{i}}-a(v) \frac{\partial v}{\partial x_{i}}\right] n_{i} g_{m}(A(u)-A(v)) d \Sigma \\
= & -\int_{\Omega} d^{\alpha}(x, t)\left[a(u) \frac{\partial u}{\partial x_{i}}-a(v) \frac{\partial v}{\partial x_{i}}\right]^{2} g_{m}^{\prime}(A(u)-A(v)) d x \leq 0,
\end{aligned}
$$

where $n=\left\{n_{i}\right\}$ is the inner unit normal vector of $\Omega$. Let $m \rightarrow \infty$. Then we have

$$
\int_{\Omega}|u(x, t)-v(x, t)| d x \leq \int_{\Omega}\left|u_{0}(x)-v_{0}(x)\right| d x .
$$


It means that the classical solutions (if there are any) of equation (1.1a) are completely determined by the initial value and free from the limitation of any boundary value condition.

Now, we will give a brief introduction of the related works on equation (1.1). Supposed that $a(u, x, t) \equiv a(u)$, when the equation is weakly degenerate, it is well known that one can impose the Dirichlet homogeneous boundary condition (1.6), one may refer to the book [2] and the references therein. When the equation is strongly degenerate, there are two ways to deal with the corresponding problem, we simply call them as the Chinese way and the international way, respectively. The Chinese way is based on the BV analysis technique, it directly answers whether (1.6) is overdetermined or not. In general, instead of the whole boundary $\partial \Omega$, only a portion of the boundary $\Sigma_{p} \subseteq \partial \Omega$ on which the trace of $u$ can be endowed in the traditional way,

$$
u(x, t)=0, \quad(x, t) \in \Sigma_{p} \times(0, T) .
$$

The representative works by Wu-Zhao [3, 4] were accomplished in the early 1980s, for later work, one may refer to [5]. While in the international way, the boundary value condition is not directly shown in the traditional way as (1.6), it is elegantly implicitly contained in family entropy inequalities. Moreover, the entropy solutions defined in the international way are only in $L^{\infty}$ space, the existence of the traditional trace (which was called the strong trace in [6]) on the boundary is not guaranteed, so the boundary value condition is satisfied in a weaker sense than that of the traditional way; one may refer to [6-13] and the references therein for details. A more explicit comment on the international way will be supplemented in Appendix 1 of our paper.

The advantage of the Chinese way lies in the fact that one can figure out on which portion of the boundary should be imposed the boundary value, whereas the rest of the boundary is free from any limitation.

Very recently, if the domain $\Omega=\mathbb{R}_{+}^{N}$ is the half space of $\mathbb{R}^{N}$, in the Chinese way, we [14] studied the initial-boundary value problem of the following equation:

$$
\frac{\partial u}{\partial t}=\Delta A(u)+\operatorname{div}(b(u)), \quad(x, t) \in \mathbb{R}_{+}^{N} \times(0, T) .
$$

We have proved that if $b_{N}^{\prime}(0)<0$, we can impose the general Dirichlet boundary condition

$$
u(x, t)=0, \quad(x, t) \in \partial \mathbb{R}_{+}^{N} \times(0, T)=\Sigma \times(0, T),
$$

which is satisfied in a particular weak sense. But if $b_{N}^{\prime}(0) \geq 0$, then no boundary condition is necessary, the solution of the equation is free from any limitation of the boundary condition.

In this paper, we continue to research how to impose a suitable homogeneous boundary condition as (1.7) in the Chinese way. Let us give the explicit formula of $\Sigma_{p}$ in (1.7) first. Let $\vec{n}=\left\{n_{i}\right\}$ be the inner unit normal vector of $\partial \Omega$. For any $\eta>0, \forall k \in \mathbb{R}$, for any given $t \in(0, T)$, denote that

$$
\begin{aligned}
& \Sigma_{1 \eta k}=\left\{x \in \Sigma, S_{\eta}(k)\left[b_{i}(0, x, t)-b_{i}(k, x, t)\right] n_{i}(x)>0\right\}, \\
& \Sigma_{2 \eta k}=\left\{x \in \Sigma, S_{\eta}(k)\left[b_{i}(0, x, t)-b_{i}(k, x, t)\right] n_{i}(x) \leq 0\right\} .
\end{aligned}
$$


Clearly, $\Sigma=\Sigma_{1 \eta k} \cup \Sigma_{2 \eta k}$, and let

$$
\Sigma_{1}=\bigcup_{\forall \eta>0, \forall k \in \mathbb{R}} \Sigma_{1 \eta k}, \quad \Sigma_{2}=\Sigma \backslash \Sigma_{1}
$$

Now, we will show that we can choose the explicit displayed formula of $\Sigma_{p}$ in (1.7) as $\Sigma_{1}$, and choose the suitable boundary condition as

$$
u(x, t)=0, \quad(x, t) \in \Sigma_{1} \times(0, T)
$$

and we will give a new kind of entropy solution to match (1.13) in a special weak sense.

Let $S_{\eta}(s)=\int_{0}^{s} h_{\eta}(\tau) d \tau$ for small $\eta>0$. Here $h_{\eta}(s)=\frac{2}{\eta}\left(1-\frac{|s|}{\eta}\right)_{+}$. The purpose of $S_{\eta}$ is to approximate the sign function $\operatorname{sgn}(s)$. Obviously $h_{\eta}(s) \in C(\mathbb{R})$, and

$$
\begin{aligned}
& h_{\eta}(s) \geq 0, \quad\left|s h_{\eta}(s)\right| \leq 1, \quad\left|S_{\eta}(s)\right| \leq 1 ; \\
& \lim _{\eta \rightarrow 0} S_{\eta}(s)=\operatorname{sgn} s, \quad \lim _{\eta \rightarrow 0} s S_{\eta}^{\prime}(s)=0 .
\end{aligned}
$$

Definition 1.1 A function $u$ is said to be the entropy solution of equation (1.1)-(1.5)-(1.13), if

1. $u \in \operatorname{BV}\left(Q_{T}\right) \cap L^{\infty}\left(Q_{T}\right)$, and there exist functions $g^{i} \in L^{2}\left(Q_{T}\right), i=1,2, \ldots, N$, such that

$$
\iint_{Q_{T}} g^{i}(x, t) \varphi(x, t) d x d t=\iint_{Q_{T}} \widehat{\gamma}^{i j}(u, x, t) \varphi(x, t) \frac{\partial u}{\partial x_{j}} d x d t
$$

where $\varphi(x, t) \in L^{2}\left(Q_{T}\right),\left(\gamma^{i j}\right)$ is the square root of $\left(a^{i j}\right)$, and

$$
\widehat{\gamma}^{i j}(u, x, t)=\int_{0}^{1} \gamma^{i j}\left(s u^{+}+(1-s) u^{-}, x, t\right) d s .
$$

2. For any $\varphi_{1}, \varphi_{2} \in C^{2}\left(\overline{Q_{T}}\right), \varphi_{1} \geq 0,\left.\nabla \varphi_{1}\right|_{\Sigma}=0,\left.\varphi_{1}\right|_{\partial \Omega \times[0, T]}=\left.\varphi_{2}\right|_{\partial \Omega \times[0, T]}$, and $\operatorname{supp} \varphi_{2}, \operatorname{supp} \varphi_{1} \subset \bar{\Omega} \times(0, T)$. For any $k \in \mathbb{R}$, any small $\eta>0$, u satisfies

$$
\begin{aligned}
& \iint_{Q_{T}}\left[I_{\eta}(u-k) \varphi_{1 t}-B_{\eta}^{i}(u, x, t, k) \varphi_{1 x_{i}}+A_{\eta}^{i j}(u, x, t, k) \varphi_{1 x_{i} x_{j}}\right. \\
& \left.\quad-S_{\eta}^{\prime}(u-k) \sum_{j=1}^{N}\left|g^{j}\right|^{2} \varphi_{1}\right] d x d t \\
& \quad+\iint_{Q_{T}} \int_{k}^{u} a_{x_{j}}^{i j}(s, x, t) S_{\eta}(s-k) d s \varphi_{1 x_{i}} d x d t \\
& \quad+S_{\eta}(k) \iint_{Q_{T}}\left[u \varphi_{2 t}-\left(b_{i}(u, x, t)-b_{i}(0, x, t)\right) \varphi_{2 x_{i}}+A^{i j}(u, x, t) \frac{\partial^{2} \varphi_{2}}{\partial x_{i} \partial x_{j}}\right. \\
& \left.\quad+\int_{0}^{u} a_{x_{j}}^{i j}(s, x, t) d s \varphi_{2 x_{i}}+\frac{\partial b_{i}(0, x, t)}{\partial x_{i}} \varphi_{2}\right] d x d t \\
& \quad+S_{\eta}(k) \int_{0}^{T} \int_{\Sigma_{1 \eta k}}\left[b_{i}(0, x, t)-b_{i}(k, x, t)\right] n_{i} \varphi_{1} d t d \sigma \geq 0 .
\end{aligned}
$$


3. The boundary value is satisfied in the sense of the trace,

$$
\left.\gamma u\right|_{\Sigma_{1 \eta k} \times(0, T)}=0 .
$$

4. The initial value is satisfied in the sense of the following equality:

$$
\lim _{t \rightarrow 0} \int_{\Omega}\left|u(x, t)-u_{0}(x)\right| d x=0
$$

Here the pairs of equal indices imply a summation from 1 up to $N$, and

$$
\begin{array}{ll}
B_{\eta}^{i}(u, x, t, k)=\int_{k}^{u} b_{i}^{\prime}(s, x, t) S_{\eta}(s-k) d s, & I_{\eta}(u-k)=\int_{0}^{u-k} S_{\eta}(s) d s, \\
A_{\eta}^{i j}(u, x, t, k)=\int_{k}^{u} a^{i j}(s, x, t) S_{\eta}(s-k) d s, & A^{i j}(u, x, t)=\int_{0}^{u} a^{i j}(s, x, t) d s .
\end{array}
$$

Let $\eta \rightarrow 0$ in (1.16). In Appendix 2 of our paper, we can see that if $u$ is the entropy solution in Definition 1.1, then it is an entropy solution as defined in [15-17].

We will prove the following theorems.

Theorem 1.2 Suppose $u_{0}(x) \in L^{\infty}(\Omega) \cap C^{2}(\Omega), A^{i j}(s, x, t)$ is $C^{3}, b_{i}(s, x, t)$ is $C^{2}$, and

$$
\begin{aligned}
& a^{i j}(0, x, t)=0, \quad(x, t) \in Q_{T}, \\
& a^{i j}(s, x, t) \xi_{i} \xi_{j}-\delta \sum_{s=1}^{N+1} \sum_{j=1}^{N}\left(a_{x_{s}}^{i j}(s, x, t) \xi_{i}\right)^{2} \geq 0 .
\end{aligned}
$$

Then equation (1.1) with the initial-boundary condition (1.5)-(1.13) has an entropy solution in the sense of Definition 1.1.

Theorem 1.3 Suppose $A^{i j}(s, x, t)$ is $C^{2}$ and $b_{i}(s, x, t)$ are $C^{1}$. Let $u, v$ be solutions of equation (1.1) with the different initial values $u_{0}(x), v_{0}(x) \in L^{\infty}(\Omega)$, respectively. Suppose there is a constant $\delta$ such that

$$
\left|\gamma^{i k}(\cdot, x, \cdot)-\gamma^{i k}(\cdot, y, \cdot)\right| \leq c|x-y|^{2+\delta}
$$

Suppose that

$$
\gamma u(x, t)=f(x, t), \quad \gamma v=g(x, t), \quad(x, t) \in \Sigma \times(0, T),
$$

and in particular

$$
\gamma u=\gamma v=0, \quad x \in \Sigma_{1}
$$

suppose that the distance function $d(x)=\operatorname{dist}(x, \Sigma)$ satisfies

$$
\left|d_{x_{i} x_{j}}\right| \leq c, \quad x \in \Omega_{\lambda}
$$


when $\lambda$ is small enough, and $\Omega_{\lambda}=\{x \in \Omega, d(x, \partial \Omega)<\lambda\}$. Then

$$
\int_{\Omega}|u(x, t)-v(x, t)| d x \leq \int_{\Omega}\left|u_{0}-v_{0}\right| d x+\text { ess } \sup _{(x, t) \in \Sigma_{2} \times(0, T)}|f(x, t)-g(x, t)| \text {, }
$$

where $(x, t) \in \mathbb{R}^{N+1}$, ess $\sup _{(x, t) \in \Sigma_{2} \times(0, T)}|f(x, t)-g(x, t)|$ is in the sense of $N$-dimensional Hausdorff measure.

Remark 1.4 If the two solutions in Theorem 1.3 are the viscous solutions of (1.1), i.e.,

$$
u=\lim _{\varepsilon \rightarrow 0} u_{\varepsilon}, \quad v=\lim _{\varepsilon \rightarrow 0} v_{\varepsilon}
$$

and $u_{\varepsilon}, v_{\varepsilon}$ is the solution of regularized problem

$$
\frac{\partial w}{\partial t}=\frac{\partial}{\partial x_{i}}\left(a^{i j}(w, x, t) \frac{\partial w}{\partial x_{j}}\right)+\varepsilon \Delta w+\frac{\partial b_{i}(w, x, t)}{\partial x_{i}}, \quad \text { in } Q_{T},
$$

with the homogeneous boundary value (1.6) and with the differential initial values $u_{0}, v_{0}$, respectively. Then $f, g$ in (1.21) is identical to 0 too. So (1.24) can be simplified to

$$
\int_{\Omega}|u(x, t)-v(x, t)| d x \leq \int_{\Omega}\left|u_{0}-v_{0}\right| d x
$$

when $u$ and $v$ are two viscous solutions of equation (1.1).

If $a^{i j}(s, x, t) \equiv a^{i j}(s), b_{i}(s, x, t) \equiv b_{i}(s)$, the above definition and the theorems had been obtained by the author in [18]. Comparing with [18], the essential improvement lies in the following two points. First, when we prove the existence of the entropy solution, we need to add the condition (1.19), and the corresponding calculation becomes more difficult and some special techniques are used. Second, due to $a^{i j}(s, x, t)$ and $b_{i}(s, x, t)$ being dependent on $(x, t) \in Q_{T}$, when we discuss the stability of the entropy solution, to ensure Kruzkov bi-variables the method still can be used successfully; not only does it take us much time to find the additional condition (1.20), but also we fortunately find the following basic but profound observation:

$$
\lim _{h \rightarrow 0} \omega_{h}^{\prime}(s) s^{2+\delta}=0
$$

where $\omega_{h}$ is the usual mollifier function. Moreover, some elegant partition techniques are ingeniously combined with Kruzkov bi-variables method, the corresponding calculation is much more complicated than that of [18] too.

The paper is arranged as follows. In the first section, we give the basic definition and the main results. In the second section, we give some basic concepts and properties of $\mathrm{BV}$ function, some lemmas are introduced and the needed estimate of the gradient of the approximate solutions is obtained, Theorem 1.2 is proved. In the third section, we will prove the stability of the entropy solutions by the Kruzkov bi-variable method. In the fourth section and the fifth section, we give a supplement to prove a lemma and a formula used before. In Appendix 1, we give a reasonable explanation of the boundary condition (1.13). In Appendix 2, we give some comments on Definition 1.1. 


\section{BV solution of the equation}

Let us first introduce the concept of BV function according to Ref. [19].

Definition 2.1 Let $\Omega \subset \mathbb{R}^{m}$ be an open set and let $f \in L^{1}(\Omega)$. Define

$$
\int_{\Omega}|D f|=\sup \left\{\int_{\Omega} f \operatorname{div} g d x: g=\left(g_{1}, g_{2}, \ldots, g_{N}\right) \in C_{0}^{1}\left(\Omega ; \mathbb{R}^{m}\right),|g(x)| \leq 1, x \in \Omega\right\},
$$

where $\operatorname{div} g=\sum_{i=1}^{m} \frac{\partial g_{i}}{\partial x_{i}}$.

Definition 2.2 A function of $f \in L^{1}(\Omega)$ is said to have a bounded variation in $\Omega$ if

$$
\int_{\Omega}|D f|<\infty
$$

We define $\operatorname{BV}(\Omega)$ as the space of all functions in $L^{1}(\Omega)$ with bounded variation.

This is equivalent to the idea that the generalized derivatives of every function in $\mathrm{BV}(\Omega)$ are regular measures on $\Omega$. Under the norm

$$
\|f\|_{\mathrm{BV}}=\|f\|_{L^{1}}+\int_{\Omega}|D f|,
$$

$\mathrm{BV}(\Omega)$ is a Banach space.

Proposition 2.3 (Semicontinuity) Let $\Omega \subseteq \mathbb{R}^{m}$ be an open set and $\left\{f_{j}\right\}$ a sequence of functions in $\mathrm{BV}(\Omega)$ which converge in $L_{\text {loc }}^{1}(\Omega)$ to a function $f$. Then

$$
\int_{\Omega}|D f| \leq \lim _{j \rightarrow \infty} \inf \int_{\Omega}\left|D f_{j}\right|
$$

Proposition 2.4 (Integration by part) Let

$$
C_{R}^{+}=\mathscr{B}(0, R) \times(0, R)=\mathscr{B}_{R} \times(0, R)
$$

and $f \in \mathrm{BV}\left(C_{R}^{+}\right)$. Then there exists a function $f^{+} \in L^{1}\left(\mathscr{B}_{R}\right)$ such that for $H_{m-1}$-almost all $y \in \mathscr{B}_{R}$,

$$
\lim _{\rho \rightarrow 0} \rho^{-m} \int_{C_{\rho}^{+}(y)}\left|f(z)-f^{+}(y)\right| d z=0 .
$$

Moreover, if $C_{R}=\mathscr{B}_{R} \times(-R, R)$, then for every $g \in C_{0}^{1}\left(C_{R} ; \mathbb{R}^{m}\right)$,

$$
\int_{C_{R}^{+}} f \operatorname{div} g d x=-\int_{C_{R}^{+}}\langle g, D f\rangle+\int_{\mathscr{B}_{R}} f^{+} g d H_{m-1},
$$

where $\mathscr{B}_{\rho}=\left\{x \in \mathbb{R}^{m} ;|x|<\rho\right\}$.

Remark 2.5 The function $f^{+}$is called the trace of $f$ on $\mathscr{B}_{R}$ and obviously

$$
f^{+}(y)=\lim _{\rho \rightarrow 0} \frac{1}{\left|C_{\rho}^{+}(y)\right|} \int_{C_{\rho}^{+}(y)} f(z) d z
$$


In our paper, we consider the solution of equation (1.1) in $\operatorname{BV}\left(Q_{T}\right)$, where $Q_{T}=\Omega \times$ $(0, T)$, and the dimension of $Q_{T}$ is $m=N+1$.

Let $\Gamma_{u}$ be the set of all jump points of $u \in \mathrm{BV}\left(Q_{T}\right), v=\left(v_{1}, v_{2}, \ldots, v_{N}, v_{N+1}\right)$ be the normal of $\Gamma_{u}$ at $X=(x, t), u^{+}(X)$ and $u^{-}(X)$ be the approximate limits of $u$ at $X \in \Gamma_{u}$ with respect to $(v, Y-X)>0$ and $(v, Y-X)<0$, respectively. For the continuous function $p(u, x, t)$ and $u \in \operatorname{BV}\left(Q_{T}\right)$, define

$$
\widehat{p}(u, x, t)=\int_{0}^{1} p\left(\tau u^{+}+(1-\tau) u^{-}, x, t\right) d \tau
$$

which is called the composite mean value of $p$. For a given $t$, we denote by $\Gamma_{u}^{t}, H^{t}$, $\left(v_{1}^{t}, \ldots, v_{N}^{t}\right)$, and $u_{ \pm}^{t}$ all jump points of $u(\cdot, t)$, the Hausdorff measure of $\Gamma_{u}^{t}$, the unit normal vector of $\Gamma_{u}^{t}$, and the asymptotic limit of $u(\cdot, t)$, respectively. Moreover, if $f(s) \in C^{1}(\mathbb{R})$, $u \in \operatorname{BV}\left(Q_{T}\right)$, then $f(u) \in \operatorname{BV}\left(Q_{T}\right)$ and

$$
\frac{\partial f(u)}{\partial x_{i}}=\widehat{f^{\prime}}(u) \frac{\partial u}{\partial x_{i}}, \quad i=1,2, \ldots, N
$$

Lemma 2.6 ([20]) Assume that $\Omega \subset \mathbb{R}^{N}$ is an open bounded set and let $f_{k}, f \in L^{q}(\Omega)$, as $k \rightarrow \infty, f_{k} \rightarrow f$ weakly in $L^{q}(\Omega), 1 \leq q<\infty$. Then

$$
\lim _{k \rightarrow \infty} \inf \left\|f_{k}\right\|_{L^{q}(\Omega)}^{q} \geq\|f\|_{L^{q(\Omega)}}^{q}
$$

The solution of our problem will be obtained as a limit point of the family $\left\{u_{\varepsilon}\right\}$ of solutions of the regularized problem

$$
\frac{\partial u}{\partial t}=\frac{\partial}{\partial x_{i}}\left(a^{i j}(u, x, t) \frac{\partial u}{\partial x_{j}}\right)+\varepsilon \Delta u+\frac{\partial b_{i}(u, x, t)}{\partial x_{i}}, \quad \text { in } Q_{T},
$$

with the compatible initial-boundary values (1.5)-(1.6).

Lemma 2.7 ([3]) Let $u_{\varepsilon}$ be the solution of equation (2.6) with initial-boundary values (1.5)(1.6). If the assumptions of Theorem 1.2 are true, then

$$
\varepsilon \int_{\Sigma}\left|\frac{\partial u_{\varepsilon}}{\partial n}\right| d \sigma \leq c_{1}+c_{2}\left(\left|\nabla u_{\varepsilon}\right|_{L^{1}(\Omega)}+\left|\frac{\partial u_{\varepsilon}}{\partial t}\right|_{L^{1}(\Omega)}\right)
$$

with constants $c_{i}, i=1,2$ independent of $\varepsilon$.

Under the assumptions of $A, b_{i}$ and $u_{0}$ in Theorem 1.2, it is well known that there is a classical solution $u_{\varepsilon}$ of the initial-boundary values problem (2.6)-(1.5)-(1.6), e.g. one may refer to Chapter 8 of [21].

We need to make some estimates for $u_{\varepsilon}$. First of all, by the maximum principle, we have

$$
\left|u_{\varepsilon}\right| \leq\left\|u_{0}\right\|_{L^{\infty}} \leq c
$$

Second, let us make the BV estimates on $u_{\varepsilon}$. 
Theorem 2.8 Let $u_{\varepsilon}$ be the solution of equation (2.6) with initial-boundary conditions (1.5)-(1.6). If the assumptions of Theorem 1.2 are true, then

$\left|\operatorname{grad} u_{\varepsilon}\right|_{L^{1}(\Omega)} \leq c$,

where $|\operatorname{grad} u|^{2}=\sum_{i=1}^{N}\left|\frac{\partial u}{\partial x_{i}}\right|^{2}+\left|\frac{\partial u}{\partial t}\right|^{2}, c$ is independent of $\varepsilon$, and independent of $t$.

Proof Differentiate (2.6) with respect to $x_{s}, s=1,2, \ldots, N, N+1, x_{N+1}=t$, and sum up for $s$ after multiplying the resulting relation by $u_{\varepsilon x_{s}} \frac{S_{\eta}\left(\left|\operatorname{grad} u_{\varepsilon}\right|\right)}{\left|\operatorname{grad} u_{\varepsilon}\right|}$. In the following, we simply denote $u_{\varepsilon}$ by $u$. Integrating over $\Omega$ yields

$$
\begin{aligned}
& \int_{\Omega} \frac{\partial u_{x_{s}}}{\partial t} u_{x_{s}} \frac{S_{\eta}(|\operatorname{grad} u|)}{|\operatorname{grad} u|} d x d x=\frac{d}{d t} \int_{\Omega} I_{\eta}(|\operatorname{grad} u|) d x, \\
& \int_{\Omega} \frac{\partial}{\partial x_{s}}\left[\frac{\partial}{\partial x_{i}}\left(a^{i j}(u, x, t) \frac{\partial u}{\partial x_{j}}\right)\right] u_{x_{s}} \frac{S_{\eta}(|\operatorname{grad} u|)}{|\operatorname{grad} u|} d x \\
& =\int_{\Omega} \frac{\partial}{\partial x_{i}}\left(a_{u}^{i j}(u, x, t) u_{x_{j}} u_{x_{s}}+a_{x_{s}}^{i j}(u, x, t) u_{x_{j}}\right) u_{x_{s}} \frac{S_{\eta}(|\operatorname{grad} u|)}{|\operatorname{grad} u|} d x \\
& \quad+\int_{\Omega} \frac{\partial}{\partial x_{i}}\left(a^{i j}(u, x, t) u_{x_{j} x_{s}}\right) u_{x_{s}} \frac{S_{\eta}(|\operatorname{grad} u|)}{|\operatorname{grad} u|} d x,
\end{aligned}
$$

and, moreover, every term in the right-hand side of (2.9) can be handled as (2.10)-(2.12), respectively,

$$
\begin{aligned}
& \int_{\Omega} \frac{\partial}{\partial x_{i}}\left(a_{u}^{i j}(u, x, t) u_{x_{j}} u_{x_{s}}\right) u_{x_{s}} \frac{S_{\eta}(|\operatorname{grad} u|)}{|\operatorname{grad} u|} d x \\
& =\int_{\Omega} \frac{\partial}{\partial x_{i}}\left(a_{u}^{i j}(u, x, t) u_{x_{j}}\right)\left[|\operatorname{grad} u| S_{\eta}(|\operatorname{grad} u|)-I_{\eta}(|\operatorname{grad} u|)\right] d x \\
& \quad-\int_{\Sigma} a_{u}^{i j}(u, x, t) u_{x_{i}} n_{j} I_{\eta}(|\operatorname{grad} u|) d \sigma \\
& \int_{\Omega} \frac{\partial}{\partial x_{i}}\left(a_{x_{s}}^{i j}(u, x, t) u_{x_{j}}\right) u_{x_{s}} \frac{S_{\eta}(|\operatorname{grad} u|)}{|\operatorname{grad} u|} d x \\
& =\int_{\Omega} \frac{\partial}{\partial x_{i}}\left(a_{x_{s}}^{i j}(u, x, t) u_{x_{j}}\right) \frac{\partial}{\partial \xi_{s}} I_{\eta}(|\operatorname{grad} u|) d x \\
& =-\int_{\Sigma} a_{x_{s}}^{i j}(u, x, t) u_{x_{j}} n_{i} \frac{\partial}{\partial \xi_{s}} I_{\eta}(|\operatorname{grad} u|) d \sigma \\
& \quad-\int_{\Omega} a_{x_{s}}^{i j}(u, x, t) u_{x_{j}} \frac{\partial^{2} I_{\eta}(|\operatorname{grad} u|)}{\partial \xi_{s} \partial \xi_{p}} u_{x_{p} x_{i}} d x \\
& \int_{\Omega} \frac{\partial}{\partial x_{i}}\left(a^{i j}(u, x, t) u_{x_{j} x_{s}}\right) u_{x_{s}} \frac{S_{\eta}(|\operatorname{grad} u|)}{|\operatorname{grad} u|} d x \\
& =\int_{\Omega} \frac{\partial}{\partial x_{i}}\left(a^{i j}(u, x, t) u_{x_{j} x_{s}}\right) \frac{\partial}{\partial \xi_{s}} I_{\eta}(|\operatorname{grad} u|) d x \\
& =-\int_{\Sigma} a^{i j}(u, x, t) u_{x_{i} x_{s}} n_{j} \frac{\partial}{\partial \xi_{s}} I_{\eta}(|\operatorname{grad} u|) d \sigma \\
& \quad-\int_{\Omega} a^{i j}(u, x, t) \frac{\partial^{2} I_{\eta}(|\operatorname{grad} u|)}{\partial \xi_{s} \partial \xi_{p}} u_{x_{s} x_{i}} u_{x_{p} x_{j}} d x
\end{aligned}
$$


where $\left\{n_{i}\right\}_{i=1}^{N}$ is the inner normal vector of $\Omega, \xi_{s}=u_{x_{s}}$. At the same time,

$$
\begin{aligned}
& \varepsilon \int_{\Omega} \Delta u_{x_{s}} u_{x_{s}} \frac{S_{\eta}(|\operatorname{grad} u|)}{|\operatorname{grad} u|} d x \\
& \quad=-\varepsilon \int_{\Sigma} \frac{\partial I_{\eta}(|\operatorname{grad} u|)}{\partial x_{i}} n_{i} d \sigma-\varepsilon \int_{\Omega} \frac{\partial^{2} I_{\eta}(|\operatorname{grad} u|)}{\partial \xi_{s} \partial \xi_{p}} u_{x_{s} x_{i}} u_{x_{p} x_{i}} d x
\end{aligned}
$$

and

$$
\begin{aligned}
& \int_{\Omega} \frac{\partial}{\partial x_{i}}\left[b_{i u}(u, x, t) u_{x_{s}}+b_{i x_{s}}(u, x, t)\right] u_{x_{s}} \frac{S_{\eta}(|\operatorname{grad} u|)}{|\operatorname{grad} u|} d x \\
& \quad=\int_{\Omega} \frac{\partial\left(b_{i u}(u, x, t) u_{x_{s}}\right)}{\partial x_{i}} u_{x_{s}} \frac{S_{\eta}(|\operatorname{grad} u|)}{|\operatorname{grad} u|} d x+\int_{\Omega} \frac{\partial b_{i x_{s}}(u, x, t)}{\partial x_{i}} u_{x_{s}} \frac{S_{\eta}(|\operatorname{grad} u|)}{|\operatorname{grad} u|} d x,
\end{aligned}
$$

here we have $b_{i x_{s}}(u, x, t)=\frac{\partial b_{i}(u, x, t)}{\partial x_{s}}, b_{i u}(u, x, t)=\frac{\partial b_{i}(u, x, t)}{\partial u}$, and

$$
\begin{aligned}
\int_{\Omega} & \frac{\partial b_{i u}(u, x, t) u_{x_{s}}}{\partial x_{i}} u_{x_{s}} \frac{S_{\eta}(|\operatorname{grad} u|)}{|\operatorname{grad} u|} d x \\
= & \int_{\Omega} \frac{\partial}{\partial x_{i}}\left(b_{i u}(u, x, t)\right)|\operatorname{grad} u| S_{\eta}(|\operatorname{grad} u|) d x+\int_{\Omega} b_{i u}(u, x, t) \frac{\partial I_{\eta}(|\operatorname{grad} u|)}{\partial x_{i}} d x \\
= & \int_{\Omega} \frac{\partial}{\partial x_{i}}\left(b_{i u}(u, x, t)\right)\left[|\operatorname{grad} u| S_{\eta}(|\operatorname{grad} u|)-I_{\eta}(|\operatorname{grad} u|)\right] d x \\
& -\int_{\Sigma} b_{i u}(u, x, t) I_{\eta}(|\operatorname{grad} u|) n_{i} d \sigma .
\end{aligned}
$$

From (2.8)-(2.14), by the assumption $a^{i j}(0, x, t)=0$, and so

$$
a_{x_{s}}^{i j}(0, x, t)=0, \quad(x, t) \in Q_{T},
$$

we have

$$
\begin{aligned}
\frac{d}{d t} \int_{\Omega} I_{\eta}(|\operatorname{grad} u|) d x \\
=\int_{\Omega} \frac{\partial}{\partial x_{i}}\left(a_{u}^{i j}(u, x, t) u_{x_{j}}\right)\left[|\operatorname{grad} u| S_{\eta}(|\operatorname{grad} u|)-I_{\eta}(|\operatorname{grad} u|)\right] d x \\
\quad-\int_{\Omega} a^{i j}(u, x, t) \frac{\partial^{2} I_{\eta}(|\operatorname{grad} u|)}{\partial \xi_{s} \partial \xi_{p}} u_{x_{s} x_{i}} u_{x_{p} x_{j}} d x-\varepsilon \int_{\Omega} \frac{\partial^{2} I_{\eta}(|\operatorname{grad} u|)}{\partial \xi_{s} \partial \xi_{p}} u_{x_{s} x_{i}} u_{x_{p} x_{i}} d x \\
\quad+\int_{\Omega} \frac{\partial}{\partial x_{i}}\left(b_{i u}(u, x, t)\right)\left[|\operatorname{grad} u| S_{\eta}(|\operatorname{grad} u|)-I_{\eta}(|\operatorname{grad} u|)\right] d x \\
\quad-\int_{\Sigma} a_{u}^{i j}(u, x, t) u_{x_{i}} n_{j} I_{\eta}(|\operatorname{grad} u|) d \sigma-\int_{\Sigma} a_{x_{s}}^{i j}(u, x, t) u_{x_{j}} u_{x_{s}} n_{i} \frac{S_{\eta}(|\operatorname{grad} u|)}{|\operatorname{grad} u|} d x \\
\quad-\int_{\Sigma} b_{i u}(u, x, t) I_{\eta}(|\operatorname{grad} u|) n_{i} d \sigma \\
\quad-\int_{\Sigma} a^{i j}(u, x, t) \frac{\partial I_{\eta}(|\operatorname{grad} u|)}{\partial x_{j}} n_{i} d \sigma-\varepsilon \int_{\Sigma} \frac{\partial I_{\eta}(|\operatorname{grad} u|)}{\partial x_{i}} n_{i} d \sigma .
\end{aligned}
$$


Now, if we set

$$
\left(\begin{array}{c}
v_{1}^{i} \\
v_{2}^{i} \\
\vdots \\
v_{N+1}^{i}
\end{array}\right)=\left(\begin{array}{cccc}
q^{11} & q^{12} & \cdots & q_{1 N+1} \\
q^{21} & q^{22} & \cdots & q_{2 N+1} \\
\vdots & & & \\
q^{N+11} & q^{N+12} & \cdots & q_{N=1 N+1}
\end{array}\right)\left(\begin{array}{c}
u_{x_{1} x_{i}} \\
u_{x_{2} x_{i}} \\
\vdots \\
u_{x_{N+1} x_{i}}
\end{array}\right)
$$

where $\left(q^{s p}\right)$ is the square root of $\left(\frac{\partial^{2} I_{\eta}}{\partial \xi_{s} \partial \xi_{p}}\right)$, then

$$
\begin{aligned}
\left|a_{x_{s}}^{i j} u_{x_{j}} \frac{\partial^{2} I_{\eta}}{\partial \xi_{s} \partial \xi_{p}} u_{x_{p} x_{i}}\right| & =\left|\left(a_{x_{1}}^{i j} u_{x_{j}}, a_{x_{2}}^{i j} u_{x_{j}}, \ldots, a_{x_{N+1}}^{i j} u_{x_{j}}\right)\left(q^{s p}\right)\left(\begin{array}{c}
v_{1}^{i} \\
v_{2}^{i} \\
\vdots \\
v_{N+1}^{i}
\end{array}\right)\right|=\left|a_{x_{s}}^{i j} u_{x_{j}} q^{s p} v_{p}^{i}\right| \\
& \leq \sum_{j=1}^{N}\left[\delta \sum_{s, p=1}^{N+1}\left(a_{x_{s}}^{i j} v_{p}^{i}\right)^{2}+\frac{1}{4 \delta} \sum_{s, p=1}^{N+1}\left(q^{s p} u_{x_{j}}\right)^{2}\right]
\end{aligned}
$$

By the assumption

$$
a^{i j}(u, x, t) \xi_{i} \xi_{j}-\delta \sum_{s=1}^{N+1} \sum_{j=1}^{N}\left(a_{x_{s}}^{i j} \xi_{j}\right)^{2} \geq 0
$$

then

$$
\begin{aligned}
& \int_{\Omega} a^{i j}(u, x, t) u_{x_{s} x_{i}} u_{x_{p} x_{j}} \frac{\partial^{2} I_{\eta}(|\operatorname{grad} u|)}{\partial \xi_{s} \partial \xi_{p}} d x-\int_{\Omega} a_{x_{s}}^{i j}(u, x, t) u_{x_{j}} \frac{\partial^{2} I_{\eta}(|\operatorname{grad} u|)}{\partial \xi_{s} \partial \xi_{p}} u_{x_{p} x_{i}} d x \\
& \quad \geq-\frac{1}{4 \delta} \int_{\Omega} \sum_{s, p=1}^{N+1} \sum_{j=1}^{N}\left(q^{s p} u_{x_{j}}\right)^{2} d x \geq-c \int_{\Omega}|\operatorname{grad} u|^{2} d x .
\end{aligned}
$$

We will use the fact that, on $\Sigma, u=0$,

$$
-b_{i u}(0, x, t) \frac{\partial u}{\partial n} n_{i}=\varepsilon \Delta u+\frac{\partial}{\partial x_{i}}\left(a^{i j}(u, x, t) \frac{\partial u}{\partial x_{j}}\right)+\frac{\partial b_{i}(u, x, t)}{\partial x_{i}},
$$

to calculate the surface integrals in (2.15). Equation (2.17) involves the derivatives on the boundary; let us give some explanation in terms of the concept of the local coordinates. Let $\delta_{0}>0$ be small enough that

$$
E^{\delta_{0}}=\left\{x \in \bar{\Omega} ; \operatorname{dist}(x, \Sigma) \leq \delta_{0}\right\} \subset \bigcup_{\tau=1}^{n} V_{\tau}
$$

where $V_{\tau}$ is a region, on which one can introduce local coordinates

$$
y_{k}=F_{\tau}^{k}(x) \quad(k=1,2, \ldots, N),\left.y_{N}\right|_{\Sigma}=0,
$$

with $F_{\tau}^{k}$ appropriately smooth and $F_{\tau}^{N}=F_{l}^{N}$, such that the $y_{N}$-axis coincides with the normal vector. Since the domain is bounded, there exists finite $V_{\tau}, \tau=1,2, \ldots, n$, such that $\bigcup_{\tau=1}^{n} V_{\tau} \supset \Sigma$. 
Using these local coordinates on $V_{\tau}, \tau=1,2, \ldots, n$, by elementary computations (refer to [3]), we obtain on $\Sigma \cap V_{\tau}$,

$$
u_{x_{i} x_{j}}=\sum_{k=1}^{N} u_{y_{N} y_{k}} F_{x_{i}}^{N} F_{x_{j}}^{k}+\sum_{k=1}^{N-1} u_{y_{N} y_{k}} F_{x_{i}}^{N} F_{x_{j}}^{k}+u_{y_{m}} F_{x_{i} x_{j}}^{m} .
$$

By this formula, what (2.17) means is clear.

Moreover, by (2.17), the surface integrals in (2.15) can be rewritten as

$$
\begin{aligned}
S= & -\left[\int_{\Sigma} b_{i u}(u, x, t) I_{\eta}(|\operatorname{grad} u|) n_{i} d \sigma+\int_{\Sigma} a^{i j}(u, x, t) \frac{\partial I_{\eta}(|\operatorname{grad} u|)}{\partial x_{j}} n_{i} d \sigma\right. \\
& \left.+\varepsilon \int_{\Sigma} \frac{\partial I_{\eta}(|\operatorname{grad} u|)}{\partial x_{i}} n_{i} d \sigma+\int_{\Sigma} a_{u}^{i j}(u, x, t) u_{x_{i}} n_{j} I_{\eta}(|\operatorname{grad} u|) d \sigma\right] \\
= & \int_{\Sigma} b_{i x_{i}}(0, x, t) \frac{I_{\eta}(|\operatorname{grad} u|)}{\frac{\partial u}{\partial n}} d \sigma-\varepsilon \int_{\Sigma}\left[\frac{\partial I_{\eta}(|\operatorname{grad} u|)}{\partial x_{i}} n_{i}-\Delta u \frac{I_{\eta}(|\operatorname{grad} u|)}{\frac{\partial u}{\partial n}}\right] d \sigma \\
& +\int_{\Sigma} a^{i j}(0, x, t)\left[\frac{\partial I_{\eta}(|\operatorname{grad} u|)}{\partial x_{i}} n_{j}-u_{x_{i} x_{j}} \frac{I_{\eta}(|\operatorname{grad} u|)}{\frac{\partial u}{\partial n}}\right] d \sigma \\
& +\int_{\Sigma} a_{x_{j}}^{i j}(0, x, t) u_{x_{j}} \frac{I_{\eta}(|\operatorname{grad} u|)}{\frac{\partial u}{\partial n}} d \sigma+\int_{\Sigma} a_{x_{s}}^{i j}(0, x, t) u_{x_{j}} u_{x_{s}} \frac{S_{\eta}(|\operatorname{grad} u|)}{|\operatorname{grad} u|} n_{i} d \sigma \\
= & \int_{\Sigma} b_{i x_{i}}(0, x, t) \frac{I_{\eta}(|\operatorname{grad} u|)}{\frac{\partial u}{\partial n}} d \sigma-\varepsilon \int_{\Sigma}\left[\frac{\partial I_{\eta}(|\operatorname{grad} u|)}{\partial x_{i}} n_{i}-\Delta u \frac{I_{\eta}(|\operatorname{grad} u|)}{\frac{\partial u}{\partial n}}\right] d \sigma .
\end{aligned}
$$

Since

$$
\left.u_{x_{N+1}}\right|_{\Sigma}=\left.u_{t}\right|_{\Sigma}=0
$$

we have

$$
\lim _{\eta \rightarrow 0} S=\int_{\Sigma} b_{i x_{i}}(0, x, t) \operatorname{sgn}\left(\frac{\partial u}{\partial n}\right) d \sigma+\varepsilon \int_{\Sigma} \operatorname{sgn}\left(\frac{\partial u}{\partial n}\right)\left(u_{x_{s} x_{i}} n_{i} n_{s}-\Delta u\right) d \sigma .
$$

Noticing that

$$
u_{x_{i} x_{j}} n_{j} n_{i}=\frac{\sum_{k=1}^{N} u_{y_{N} y_{k}} F_{x_{i}}^{N} F_{x_{j}}^{k} F_{x_{j}}^{N} F_{x_{i}}^{N}}{\left|\operatorname{grad} F^{N}\right|^{2}}+\sum_{k=1}^{N-1} u_{y_{N} y_{k}} F_{x_{i}}^{k} F_{x_{j}}^{N}+\frac{u_{y_{m}} F_{x_{i} x_{j}}^{m} F_{x_{j}}^{N} F_{x_{i}}^{N}}{\left|\operatorname{grad} F^{N}\right|^{2}}
$$

in which $F^{k}=F_{\tau}^{k}$, by the fact that the normal vector is

$$
\vec{n}=\left(\frac{\partial F^{N}}{\partial x_{1}}, \ldots, \frac{\partial F^{N}}{\partial x_{N}}\right)=\operatorname{grad} F^{N},
$$

we have

$$
u_{x_{i} x_{j}} n_{j} n_{i}-\Delta u=u_{y_{m}}\left(\frac{F_{x_{i} x_{j}}^{m} F_{x_{j}}^{N} F_{x_{i}}^{N}}{\left|\operatorname{grad} F^{N}\right|^{2}}-F_{x_{i} x_{i}}^{m}\right)
$$

Using Lemma 2.7, one is able to deduce that $\lim _{\eta \rightarrow 0} S$ can be estimated by $|\operatorname{grad} u|_{L_{1}(\Omega)}$. 
Thus, letting $\eta \rightarrow 0$ in (2.15), and noticing that

$$
\lim _{\eta \rightarrow 0}\left[|\operatorname{grad} u| S_{\eta}(|\operatorname{grad} u|)-I_{\eta}(|\operatorname{grad} u|)\right]=0
$$

using the fact of that $\lim _{\eta \rightarrow 0} S$ can be estimated by $|\operatorname{grad} u|_{L_{1}(\Omega)}$, we have

$$
\frac{d}{d t} \int_{\Omega}|\operatorname{grad} u| d x \leq c_{1}+c_{2} \int_{\Omega}|\operatorname{grad} u| d x
$$

by the well-known Gronwall lemma, we have

$$
\int_{\Omega}|\operatorname{grad} u| d x d t \leq c
$$

By (2.19), it is easy to show that

$$
\iint_{Q_{T}} a^{i j}(u, x, t) u_{x_{i}} u_{x_{j}} d x d t \leq c .
$$

Now we put back the solution of equation (2.6) as $u_{\varepsilon}$. Then by (2.19)-(2.20), there exist a subsequence $\left\{u_{\varepsilon_{n}}\right\}$ of $u_{\varepsilon}$ and a function $u \in \operatorname{BV}\left(Q_{T}\right) \cap L^{\infty}\left(Q_{T}\right)$ such that $u_{\varepsilon_{n}} \rightarrow u$ a.e. on $Q_{T}$, we can simply denote this subsequence as $\{\varepsilon\}$ itself; there exist functions $g^{i} \in L^{2}\left(Q_{T}\right)$ and a subsequence of $\{\varepsilon\}$, such that, when $\varepsilon \rightarrow 0$,

$$
\widehat{\gamma^{i j}} \frac{\partial u_{\varepsilon}}{\partial x_{j}} \rightarrow g^{i}, \quad \text { in } L^{2}\left(Q_{T}\right)
$$

Proof of Theorem 1.2 We now prove that $u$ is a generalized solution of (1.1)-(1.5)-(1.13). Let $\varphi \in C^{2}\left(\overline{Q_{T}}\right), \varphi_{1} \geq 0, \operatorname{supp} \varphi \subset \bar{\Omega} \times(0, T),\left.\nabla \varphi_{1}\right|_{\Omega}=0$, and $\left\{n_{i}\right\}$ be the inner normal vector of $\Omega$. Multiply equation (2.6) by $\varphi_{1} S_{\eta}\left(u_{\varepsilon}-k\right)$, and integrate over $Q_{T}$, to obtain

$$
\begin{aligned}
& \iint_{Q_{T}} \frac{\partial u_{\varepsilon}}{\partial t} \varphi_{1} S_{\eta}\left(u_{\varepsilon}-k\right) d x d t \\
& =\iint_{Q_{T}} \frac{\partial}{\partial x_{i}}\left(a^{i j}\left(u_{\varepsilon}\right) \frac{\partial u_{\varepsilon}}{\partial x_{j}}\right) \varphi_{1} S_{\eta}\left(u_{\varepsilon}-k\right) d x d t \\
& \quad+\varepsilon \iint_{Q_{T}} \Delta u_{\varepsilon} \varphi_{1} S_{\eta}\left(u_{\varepsilon}-k\right) d x d t+\iint_{Q_{T}} \frac{\partial b_{i}\left(u_{\varepsilon}, x, t\right)}{\partial x_{i}} \varphi_{1} S_{\eta}\left(u_{\varepsilon}-k\right) d x d t .
\end{aligned}
$$

Let us calculate every term in (2.21) by the partial integration method. We have

$$
\begin{aligned}
& \iint_{Q_{T}} \frac{\partial u_{\varepsilon}}{\partial t} \varphi_{1} S_{\eta}\left(u_{\varepsilon}-k\right) d x d t=-\iint_{Q_{T}} I_{\eta}\left(u_{\varepsilon}-k\right) \varphi_{1 t} d x d t, \\
& \varepsilon \iint_{Q_{T}} \Delta u_{\varepsilon} \varphi_{1} S_{\eta}\left(u_{\epsilon}-k\right) d x d t \\
& \quad=-\varepsilon \int_{0}^{T} \int_{\Sigma} \nabla u_{\varepsilon} \cdot \vec{n} \varphi_{1} S_{\eta}\left(u_{\epsilon}-k\right) d t d \sigma \\
& \quad-\varepsilon \iint_{Q_{T}} \nabla u_{\varepsilon}\left[S_{\eta}\left(u_{\varepsilon}-k\right) \nabla \varphi_{1}+\varphi_{1} S_{\eta}^{\prime}\left(u_{\varepsilon}-k\right) \nabla u_{\varepsilon}\right] d x d t
\end{aligned}
$$




$$
\begin{aligned}
& =\varepsilon S_{\eta}(k) \int_{0}^{T} \int_{\Sigma} \nabla u_{\varepsilon} \cdot \vec{n} \varphi_{1} d t d \sigma-\varepsilon \iint_{Q_{T}} \nabla u_{\varepsilon} S_{\eta}\left(u_{\varepsilon}-k\right) \nabla \varphi_{1} d x d t \\
& \quad-\varepsilon \iint_{Q_{T}}\left|\nabla u_{\varepsilon}\right|^{2} S_{\eta}^{\prime}\left(u_{\varepsilon}-k\right) \varphi_{1} d x d t \\
& \iint_{Q_{T}} \frac{\partial}{\partial x_{i}}\left(a^{i j}\left(u_{\varepsilon}, x, t\right) \frac{\partial u_{\varepsilon}}{\partial x_{j}}\right) \varphi_{1} S_{\eta}\left(u_{\varepsilon}-k\right) d x d t \\
& =S_{\eta}(k) \int_{0}^{T} \int_{\Sigma} a^{i j}\left(u_{\varepsilon}, x, t\right) \frac{\partial u_{\varepsilon}}{\partial x_{j}} n_{i} \varphi_{1} d t d \sigma \\
& \quad-\iint_{Q_{T}} a^{i j}\left(u_{\varepsilon}, x, t\right) \frac{\partial u_{\varepsilon}}{\partial x_{j}}\left[S_{\eta}\left(u_{\varepsilon}-k\right) \varphi_{1 x_{i}}+\varphi_{1} S_{\eta}^{\prime}\left(u_{\varepsilon}-k\right) u_{\varepsilon x_{i}}\right] d x d t \\
& =S_{\eta}(k) \int_{0}^{T} \int_{\Sigma} a^{i j}\left(u_{\varepsilon}, x, t\right) \frac{\partial u_{\varepsilon}}{\partial x_{j}} n_{i} \varphi_{1} d t d \sigma-\iint_{Q_{T}} a^{i j}\left(u_{\varepsilon}, x, t\right) \frac{\partial u_{\varepsilon}}{\partial x_{j}} S_{\eta}\left(u_{\varepsilon}-k\right) \varphi_{1 x_{i}} d x d t \\
& \quad-\iint_{Q_{T}} a^{i j}\left(u_{\varepsilon}, x, t\right) u_{\varepsilon x_{i}} u_{\varepsilon x_{j}} S_{\eta}^{\prime}\left(u_{\varepsilon}-k\right) \varphi_{1} d x d t
\end{aligned}
$$

and

$$
\begin{aligned}
& -\iint_{Q_{T}} a^{i j}\left(u_{\varepsilon}, x, t\right) \frac{\partial u_{\varepsilon}}{\partial x_{j}} S_{\eta}\left(u_{\varepsilon}-k\right) \varphi_{1 x_{i}} d x d t \\
& =\iint_{Q_{T}} \int_{k}^{u_{\varepsilon}} a_{x_{j}}^{i j}(s, x, t) S_{\eta}(s-k) d s \varphi_{1 x_{i}} d x d t \\
& \quad+\iint_{Q_{T}} A_{\eta}^{i j}\left(u_{\varepsilon}, x, t, k\right) \varphi_{1 x_{i} x_{j}} d x d t+\int_{0}^{T} \int_{\Sigma} A_{\eta}^{i j}\left(u_{\varepsilon}, k\right) \varphi_{1 x_{i}} n_{j} d t d \sigma, \\
& \iint_{Q_{T}} \frac{\partial b_{i}\left(u_{\varepsilon}, x, t\right)}{\partial x_{i}} \varphi_{1} S_{\eta}\left(u_{\varepsilon}-k\right) d x d t \\
& =-\int_{0}^{T} \int_{\Sigma}\left[b_{i}\left(u_{\varepsilon}, x, t\right)-b(k, x, t)\right] n_{i} \varphi_{1} S_{\eta}\left(u_{\varepsilon}-k\right) d t d \sigma \\
& \quad-\iint_{Q_{T}}\left[b_{i}\left(u_{\varepsilon}, x, t\right)-b_{i}(k, x, t)\right]\left[\frac{\partial \varphi_{1}}{\partial x_{i}} S_{\eta}\left(u_{\varepsilon}-k\right)+\varphi_{1} S_{\eta}^{\prime}\left(u_{\varepsilon}-k\right) \frac{\partial u_{\varepsilon}}{\partial x_{i}}\right] d x d t \\
& =-S_{\eta}(k) \int_{0}^{T} \int_{\Sigma} \varphi_{1}\left[b_{i}(0, x, t)-b_{i}(k, x, t)\right] n_{i} d \sigma d t \\
& \quad-\iint_{Q_{T}} B_{\eta}^{i}\left(u_{\varepsilon}, x, t, k\right) \varphi_{1 x_{i}} d x d t .
\end{aligned}
$$

From (2.21)-(2.26), we have

$$
\begin{aligned}
& \iint_{Q_{T}} I_{\eta}\left(u_{\varepsilon}-k\right) \varphi_{1 t} d x d t+\iint_{Q_{T}} A_{\eta}^{i j}\left(u_{\varepsilon}, x, t, k\right) \varphi_{1 x_{i} x_{j}} d x d t-\iint_{Q_{T}} B_{\eta}^{i}\left(u_{\varepsilon}, x, t, k\right) \varphi_{1 x_{i}} d x d t \\
& \quad-\varepsilon \iint_{Q_{T}} \nabla u_{\varepsilon} \cdot \nabla \varphi_{1} S_{\eta}\left(u_{\varepsilon}-k\right) d x d t-\varepsilon \iint_{Q_{T}}\left|\nabla u_{\varepsilon}\right|^{2} S_{\eta}^{\prime}\left(u_{\varepsilon}-k\right) \varphi_{1} d x d t \\
& \quad-\iint_{Q_{T}} a^{i j}\left(u_{\varepsilon}, x, t\right) u_{\varepsilon x_{i}} u_{\varepsilon x_{j}} S_{\eta}^{\prime}\left(u_{\varepsilon}-k\right) \varphi_{1} d x d t \\
& \quad+\iint_{Q_{T}} \int_{k}^{u_{\varepsilon}} a_{x_{j}}^{i j}(s, x, t) S_{\eta}(s-k) d s \varphi_{1 x_{i}} d x d t
\end{aligned}
$$




$$
\begin{aligned}
& +S_{\eta}(k) \int_{0}^{T} \int_{\Sigma} \frac{\partial}{\partial x_{i}}\left(a^{i j}\left(u_{\varepsilon}, x, t\right)\right) n_{i} u_{\varepsilon x_{j}} \varphi_{1} d t d \sigma+S_{\eta}(k) \int_{0}^{T} \int_{\Sigma} A_{\eta}^{i j}(0, x, t, k) \varphi_{1 x_{i}} n_{j} d t d \sigma \\
& +\varepsilon S_{\eta}(k) \int_{0}^{T} \int_{\Sigma} \nabla u_{\varepsilon} \cdot \vec{n} \varphi_{1} d t d \sigma+S_{\eta}(k) \int_{0}^{T} \int_{\Sigma_{1 \eta k}}\left[b_{i}(0, x, t)-b_{i}(k, x, t)\right] n_{i} \varphi_{1} d t d \sigma \\
& +S_{\eta}(k) \int_{0}^{T} \int_{\Sigma_{2 \eta k}}\left[b_{i}(0, x, t)-b_{i}(k, x, t)\right] n_{i} \varphi_{1} d t d \sigma=0 .
\end{aligned}
$$

Taking $\varphi_{2} \in C^{2}\left(\bar{Q}_{T}\right),\left.\varphi_{1}\right|_{\partial \Omega \times[0, T]}=\left.\varphi_{2}\right|_{\partial \Omega \times[0, T]}, \operatorname{supp} \varphi_{2} \subset \bar{\Omega} \times(0, T)$,

$$
\begin{aligned}
& S_{\eta}(k) \int_{0}^{T} \int_{\Sigma} a^{i j}\left(u_{\varepsilon}, x, t\right) n_{i} \frac{\partial u_{\varepsilon}}{\partial x_{j}} \varphi_{1} d t d \sigma+\varepsilon S_{\eta}(k) \int_{0}^{T} \int_{\Sigma} \nabla u_{\varepsilon} \cdot \vec{n} \varphi_{1} d t d \sigma \\
& =S_{\eta}(k)\left\{-\varepsilon \iint_{Q_{T}} \frac{\partial u_{\varepsilon}}{\partial x_{i}} \frac{\partial \varphi_{2}}{\partial x_{i}} d x d t-\iint_{Q_{T}} a^{i j}\left(u_{\varepsilon}, x, t\right) \frac{\partial u_{\varepsilon}}{\partial x_{j}} \varphi_{2 x_{i}} d x d t\right. \\
& +\iint_{Q_{T}} \frac{\partial b_{i}(0, x, t)}{\partial x_{i}} \varphi_{2} d x d t-\iint_{Q_{T}}\left(b_{i}\left(u_{\varepsilon}, x, t\right)-b_{i}(0, x, t)\right) \frac{\partial \varphi_{2}}{\partial x_{i}} d x d t \\
& \left.+\iint_{Q_{T}} u_{\varepsilon} \frac{\partial \varphi_{2}}{\partial t} d x d t-\int_{0}^{T} \int_{\Sigma}\left[b_{i}(0, x, t)-b_{i}(0, x, t)\right] n_{i} \varphi_{2} d t d \sigma\right\} \\
& \iint_{Q_{T}} a^{i j}\left(u_{\varepsilon}, x, t\right) \varphi_{2 x_{i}} \frac{\partial u_{\varepsilon}}{\partial x_{j}} d x d t \\
& =-\int_{0}^{T} \int_{\Sigma} A^{i j}(0, x, t) \varphi_{2 x_{i}} n_{j} d t d \sigma \\
& -\iint_{Q_{T}} A^{i j}\left(u_{\varepsilon}, x, t\right) \varphi_{2 x_{i} x_{j}} d x d t-\iint_{Q_{T}} \int_{0}^{u_{\varepsilon}} a_{x_{j}}^{i j}(s, x, t) d s \varphi_{2 x_{i}} d x d t \\
& =-\iint_{Q_{T}} A^{i j}\left(u_{\varepsilon}, x, t\right) \varphi_{2 x_{i} x_{j}} d x d t-\iint_{Q_{T}} \int_{0}^{u_{\varepsilon}} a_{x_{j}}^{i j}(s, x, t) d s \varphi_{2 x_{i}} d x d t .
\end{aligned}
$$

For $\left.\nabla \varphi_{1}\right|_{\Sigma}=0$, and by $a^{i j}(0, x, t)=0$, from (2.27)-(2.29), we have

$$
\begin{aligned}
& \iint_{Q_{T}} I_{\eta}\left(u_{\varepsilon}-k\right) \varphi_{1 t} d x d t+\iint_{Q_{T}} A_{\eta}^{i j}\left(u_{\varepsilon}, x, t, k\right) \varphi_{1 x_{i} x_{j}} d x d t-\iint_{Q_{T}} B_{\eta}^{i}\left(u_{\varepsilon}, x, t, k\right) \varphi_{1 x_{i}} d x d t \\
& +\iint_{Q_{T}} \int_{0}^{u_{\varepsilon}} a_{x_{j}}^{i j}(s, x, t) S_{\eta}(s-k) d s \varphi_{1 x_{i}} d x d t \\
& +S_{\eta}(k)\left[-\varepsilon \iint_{Q_{T}} \frac{\partial u_{\varepsilon}}{\partial x_{i}} \frac{\partial \varphi_{2}}{\partial x_{i}} d x d t+\iint_{Q_{T}} A^{i j}\left(u_{\varepsilon}, x, t\right) \varphi_{2 x_{i} x_{j}} d x d t\right. \\
& +\iint_{Q_{T}} \frac{\partial b_{i}(0, x, t)}{\partial x_{i}} \varphi_{2} d x d t \\
& \left.-\iint_{Q_{T}}\left[b_{i}\left(u_{\varepsilon}, x, t\right)-b_{i}(0, x, t)\right] \frac{\partial \varphi_{2}}{\partial x_{i}} d x d t+\iint_{Q_{T}} u_{\varepsilon} \frac{\partial \varphi_{2}}{\partial t} d x d t\right] \\
& +S_{\eta}(k) \iint_{Q_{T}} \int_{0}^{u_{\varepsilon}} a_{x_{j}}^{i j}(s, x, t) d s \varphi_{2 x_{i}} d x d t \\
& -\varepsilon \iint_{Q_{T}} \nabla u_{\varepsilon} \cdot \nabla \varphi_{1} S_{\eta}\left(u_{\varepsilon}-k\right) d x d t-\iint_{Q_{T}} a^{i j}\left(u_{\varepsilon}, x, t\right) u_{\varepsilon x_{i}} u_{\varepsilon x_{j}} S_{\eta}^{\prime}\left(u_{\varepsilon}-k\right) \varphi_{1} d x d t \\
& +S_{\eta}(k) \int_{0}^{T} \int_{\Sigma_{1 \eta k}}\left[\left(b_{i}(0)-b_{i}(k)\right] n_{i} \varphi_{1} d t d \sigma \geq 0\right.
\end{aligned}
$$


By Lemma 2.6,

$$
\begin{gathered}
\lim \inf _{\varepsilon \rightarrow 0} \iint_{Q_{T}} S_{\eta}^{\prime}\left(u_{\varepsilon}-k\right) a^{i j}\left(u_{\varepsilon}, x, t\right) \frac{\partial u_{\varepsilon}}{\partial x_{i}} \frac{\partial u_{\varepsilon}}{\partial x_{j}} \varphi_{1} d x d t \\
\geq \sum_{i=1}^{N} \iint_{Q_{T}}\left|g^{i}\right|^{2} S_{\eta}^{\prime}(u-k) \varphi_{1} d x d t .
\end{gathered}
$$

Let $\varepsilon \rightarrow 0$ in (2.30). By (2.31), we get (1.16) and (1.17) is naturally concealed in the limiting process.

The proof of $(1.18)$ is similar to that in $[15,22]$, we omit the details here.

\section{Proof of Theorem 1.3}

Lemma 3.1 Let u be a solution of equation (1.1). Then

$$
\int_{u^{-}}^{u^{+}} \gamma^{i j}(s, x, t) d s \cdot v_{i}=0, \quad \text { a.e. }(x, t) \text { on } \Gamma_{u}, j=1,2, \ldots, N
$$

is true in the sense of Hausdorff measure $H_{N}\left(\Gamma_{u}\right)$.

The proof is given in Section 5 as follows.

Proof of Theorem 1.3 Let $u, v$ be two entropy solutions of equation (1.1) with different initial values

$$
u(x, 0)=u_{0}(x), \quad v(x, 0)=v_{0}(x)
$$

and with the same homogeneous boundary value $\gamma u(x, t)=\gamma v(x, t)=0,(x, t) \in \Sigma_{1} \times(0, T)$.

By Definition 1.1, for any $\varphi_{1}, \varphi_{2} \in C^{2}\left(\overline{Q_{T}}\right), \varphi_{1} \geq 0,\left.\varphi_{1}\right|_{\partial \Omega \times[0, T]}=\left.\varphi_{2}\right|_{\partial \Omega \times[0, T]}, \operatorname{supp} \varphi_{2}$, $\operatorname{supp} \varphi_{1} \subset \bar{\Omega} \times(0, T), \eta>0, k, l \in \mathbb{R}$, we have

$$
\begin{aligned}
& \iint_{Q_{T}}\left[I_{\eta}(u-k) \varphi_{1 t}-B_{\eta}^{i}(u, x, t, k) \varphi_{1 x_{i}}+A_{\eta}^{i j}(u, x, t, k) \varphi_{1 x_{i} x_{j}}\right. \\
& \left.\quad-S_{\eta}^{\prime}(u-k) \sum_{i=1}^{N}\left|g^{i}(u, x, t)\right|^{2} \varphi_{1}\right] d x d t+\iint_{Q_{T}} \int_{k}^{u} a_{x_{j}}^{i j}(s, x, t) S_{\eta}(s-k) d s \varphi_{1 x_{i}} d x d t \\
& \quad+S_{\eta}(k)\left[b_{i}(0, x, t)-b_{i}(k, x, t)\right] \int_{0}^{T} \int_{\Sigma_{1 \eta k}} \varphi_{1} n_{i} d t d \sigma \\
& \quad+S_{\eta}(k) \iint_{Q_{T}}\left[u \varphi_{2 t}-\left(b_{i}(u, x, t)-b_{i}(0, x, t)\right) \varphi_{2 x_{i}}\right. \\
& \left.\quad+A^{i j}(u, x, t) \varphi_{2 x_{i} x_{j}}+\frac{\partial b_{i}(0, x, t)}{\partial x_{i}} \varphi_{2}+\int_{0}^{u} a_{x_{j}}^{i j}(s, x, t) d s \varphi_{2 x_{i}}\right] d x d t \geq 0, \\
& \iint_{Q_{T}}\left[I_{\eta}(v-l) \varphi_{1 \tau}-B_{\eta}^{i}(v, y, \tau, l) \varphi_{1 y_{i}}+A_{\eta}^{i j}(v, y, \tau, l) \varphi_{1 y_{i} y_{j}}\right. \\
& \left.\quad-S_{\eta}^{\prime}(v-l) \sum_{i=1}^{N}\left|g^{i}(v, y, \tau)\right|^{2} \varphi_{1}\right] d y d \tau+\iint_{Q_{T}}^{v} \int_{l}^{v} a_{y_{j}}^{i j}(s, y, \tau) S_{\eta}(s-l) d s \varphi_{1 y_{i}} d x d t
\end{aligned}
$$




$$
\begin{aligned}
& +S_{\eta}(l)\left[b_{i}(0, y, \tau)-b_{i}(l, y, \tau)\right] \int_{0}^{T} \int_{\Sigma_{1 \eta k}} \varphi_{1} n_{i} d \tau d \sigma \\
& +S_{\eta}(l) \iint_{Q_{T}}\left[v \varphi_{2 \tau}-\left(b_{i}(v, y, \tau)-b_{i}(0, y, \tau)\right) \varphi_{2 y_{i}}\right. \\
& \left.+A^{i j}(v, y, \tau) \varphi_{2 y_{i} y_{j}}+\frac{\partial b_{i}(0, y, \tau)}{\partial y_{i}} \varphi_{2}+\int_{0}^{v} a_{y_{j}}^{i j}(s, y, \tau) d s \varphi_{2 y_{i}}\right] d y d \tau \geq 0 .
\end{aligned}
$$

Especially, if $\varphi_{1} \in C_{0}^{2}\left(Q_{T}\right), \varphi_{2} \equiv 0$, we have

$$
\begin{aligned}
& \iint_{Q_{T}}\left[I_{\eta}(u-k) \varphi_{1 t}-B_{\eta}^{i}(u, x, t, k) \varphi_{1 x_{i}}+A_{\eta}^{i j}(u, x, t, k) \varphi_{1 x_{i} x_{j}}-S_{\eta}^{\prime}(u-k) \sum_{i=1}^{N}\left|g^{i}(u, x, t)\right|^{2} \varphi_{1}\right. \\
& \left.\quad+\iint_{Q_{T}} \int_{k}^{u} a_{x_{j}}^{i j}(s, x, t) S_{\eta}(s-k) d s \varphi_{1 x_{i}} d x d t\right] d x d t \geq 0 \\
& \iint_{Q_{T}}\left[I_{\eta}(v-l) \varphi_{1 \tau}-B_{\eta}^{i}(v, y, \tau, l) \varphi_{1 y_{i}}+A_{\eta}^{i j}(v, y, \tau, l) \varphi_{1 y_{i} y_{j}}-S_{\eta}^{\prime}(v-l) \sum_{i=1}^{N}\left|g^{i}\right|^{2}(v, y, \tau) \varphi_{1}\right. \\
& \left.\quad+\iint_{Q_{T}} \int_{l}^{v} a_{y_{j}}^{i j}(s, y, \tau) S_{\eta}(s-l) d s \varphi_{1 y_{i}} d x d t\right] d y d \tau \geq 0
\end{aligned}
$$

Let $\psi(x, t, y, \tau)=\phi(x, t) j_{h}(x-y, t-\tau)$. Here $\phi(x, t) \geq 0, \phi(x, t) \in C_{0}^{\infty}\left(Q_{T}\right)$, and

$$
\begin{aligned}
& j_{h}(x-y, t-\tau)=\omega_{h}(t-\tau) \prod_{i=1}^{N} \omega_{h}\left(x_{i}-y_{i}\right), \\
& \omega_{h}(s)=\frac{1}{h} \omega\left(\frac{s}{h}\right), \quad \omega(s) \in C_{0}^{\infty}(R), \quad \omega(s) \geq 0, \\
& \omega(s)=0 \quad \text { if }|s|>1, \quad \int_{-\infty}^{\infty} \omega(s) d s=1 .
\end{aligned}
$$

Moreover, for any given positive constant $\delta$,

$$
\lim _{h \rightarrow 0} \omega_{h}^{\prime}(s) s^{2+\delta}=0
$$

Then we choose $k=v(y, \tau), l=u(x, t), \varphi_{1}=\psi(x, t, y, \tau)$ in (3.4), (3.5), integrate over $Q_{T}$, respectively, plus them together and get the following inequality:

$$
\begin{aligned}
& \iint_{Q_{T}} \iint_{Q_{T}}\left[I_{\eta}(u-v)\left(\psi_{t}+\psi_{\tau}\right)-\left(B_{\eta}^{i}(u, x, t, v) \psi_{x_{i}}+B_{\eta}^{i}(v, y, \tau, u) \psi_{y_{i}}\right)\right. \\
& \left.\quad+A_{\eta}^{i j}(u, x, t, v) \psi_{x_{i} x_{j}}+A_{\eta}^{i j}(v, y, \tau, u) \psi_{y_{i} y_{j}}\right] \\
& \quad+\int_{v}^{u} a_{x_{j}}^{i j}(s, x, t) S_{\eta}(s-v) d s \psi_{x_{i}}+\int_{u}^{v} a_{y_{j}}^{i j}(s, y, \tau) S_{\eta}(s-u) d s \psi_{y_{i}} \\
& \quad-S_{\eta}^{\prime}(u-v)\left(\sum_{i=1}^{N}\left|g^{i}(u, x, t)\right|^{2}+\sum_{i=1}^{N}\left|g^{i}(v, y, \tau)\right|^{2}\right) \psi d x d t d y d \tau \geq 0 .
\end{aligned}
$$


Clearly,

$$
\begin{aligned}
& \frac{\partial j_{h}}{\partial t}+\frac{\partial j_{h}}{\partial \tau}=0, \quad \frac{\partial j_{h}}{\partial x_{i}}+\frac{\partial j_{h}}{\partial y_{i}}=0, \quad i=1, \ldots, N ; \\
& \frac{\partial \psi}{\partial t}+\frac{\partial \psi}{\partial \tau}=\frac{\partial \phi}{\partial t} j_{h}, \quad \frac{\partial \psi}{\partial x_{i}}+\frac{\partial \psi}{\partial y_{i}}=\frac{\partial \phi}{\partial x_{i}} j_{h} .
\end{aligned}
$$

Noticing that

$$
\lim _{\eta \rightarrow 0} B_{\eta}^{i}(u, x, t, v)=\operatorname{sgn}(u-v)\left(b_{i}(u, x, t)-b_{i}(v, x, t)\right),
$$

and

$$
\lim _{\eta \rightarrow 0} B_{\eta}^{i}(v, y, \tau, u)=\operatorname{sgn}(v-u)\left(b_{i}(v, y, \tau)-b_{i}(u, y, \tau)\right),
$$

as $\eta \rightarrow 0$, we have

$$
\begin{aligned}
& \lim _{\eta \rightarrow 0} \iint_{Q_{T}} \iint_{Q_{T}}\left[B_{\eta}^{i}(u, x, t, v) \psi_{x_{i}}+B_{\eta}^{i}(v, y, \tau, u) \psi_{y_{i}}\right] d x d t d y d \tau \\
& =\iint_{Q_{T}} \iint_{Q_{T}} \operatorname{sgn}(u-v)\left[b_{i}(u, x, t)-b_{i}(v, y, \tau)\right] \phi_{x_{i}} j_{h} d x d t d y d \tau \\
& \quad+\iint_{Q_{T}} \iint_{Q_{T}} \operatorname{sgn}(u-v)\left[b_{i}(v, y, \tau)-b_{i}(v, x, t)\right] \phi_{x_{i}} j_{h} d x d t d y d \tau,
\end{aligned}
$$

and as $h \rightarrow 0$, we have

$$
\begin{gathered}
\lim _{h \rightarrow 0} \lim _{\eta \rightarrow 0} \iint_{Q_{T}} \iint_{Q_{T}}\left[B_{\eta}^{i}(u, x, t, v) \psi_{x_{i}}+B_{\eta}^{i}(v, y, \tau, u) \psi_{y_{i}}\right] d x d t d y d \tau \\
\quad=\iint_{Q_{T}} \operatorname{sgn}(u-v)\left[b_{i}(u, x, t)-b_{i}(v, x, t)\right] \phi_{x_{i}} d x d t .
\end{gathered}
$$

For simplicity, we denote

$$
\begin{aligned}
& I_{3}=\iint_{Q_{T}} \iint_{Q_{T}}\left[A_{\eta}^{i j}(u, x, t, v) \psi_{x_{i} x_{j}}+A_{\eta}^{i j}(v, y, \tau, u) \psi_{y_{i} y_{j}}\right] d x d t d y d \tau, \\
& I_{4}=-\iint_{Q_{T}} \iint_{Q_{T}} S_{\eta}^{\prime}(u-v) \sum_{n=1}^{N}\left(\left|g^{j}(u, x, t)\right|^{2}+\left|g^{j}(v, y, \tau)\right|^{2}\right) \psi d x d t d y d \tau, \\
& I_{5}=\iint_{Q_{T}} \iint_{Q_{T}} \int_{v}^{u} a_{x_{j}}^{i j} S_{\eta}(s-v) d s \phi_{x_{j}} j_{h} d x d t d y d \tau, \\
& I_{6}=\iint_{Q_{T}} \iint_{Q_{T}} \int_{u}^{v} a_{y_{j}}^{i j} S_{\eta}(s-u) d s \phi_{x_{i}} j_{h} d x d t d y d \tau, \\
& I_{7}=\iint_{Q_{T}} \iint_{Q_{T}} \int_{v}^{u} a_{x_{j}}^{i j} S_{\eta}(s-v) d s \phi j_{h x_{i}} d x d t d y d \tau, \\
& I_{8}=-\iint_{Q_{T}} \iint_{Q_{T}} \int_{u}^{v} a_{y_{j}}^{i j} S_{\eta}^{\prime}(s-u) d s u_{x_{i}} \phi j_{h} d x d t d y d \tau .
\end{aligned}
$$


Then we have

$$
\begin{aligned}
I_{3}= & \iint_{Q_{T}} \iint_{Q_{T}}\left[A_{\eta}^{i j}(u, x, t, v)\left(\phi_{x_{i} x_{j}} j_{h}+2 \phi_{x_{i}} j_{h x_{j}}+\phi j_{h x_{i} x_{j}}\right)\right. \\
& \left.+A_{\eta}^{i j}(v, y, \tau, u) \phi j_{h y_{i} y_{j}}\right] d x d y d \tau d t \\
= & \iint_{Q_{T}} \iint_{Q_{T}}\left[A_{\eta}^{i j}(u, x, t, v)\left(\phi_{x_{i} x_{j}} j_{h}+\phi_{x_{i}} j_{h x_{j}}\right)-A_{\eta}^{i j}(v, y, \tau, u) \phi_{x_{j}} j_{h x_{j}}\right. \\
& \left.-\partial_{x_{j}} A_{\eta}^{i j}(u, x, t, v) \phi j_{h x_{i}}-\partial_{x_{i}} A_{\eta}^{i j}(v, y, \tau, u) \phi j_{h x_{j}}\right] d x d y d t d \tau \\
= & \iint_{Q_{T}} \iint_{Q_{T}}\left[A_{\eta}^{i j}(u, x, t, v)\left(\phi_{x_{i} x_{j}} j_{h}+\phi_{x_{i}} j_{h x_{j}}\right)-A_{\eta}^{i j}(v, y, \tau, u) \phi_{x_{i}} j_{h x_{j}}\right] d x d t d y d \tau \\
& -\iint_{Q_{T}} \iint_{Q_{T}}\left[\int_{0}^{1} a^{i j}\left(\sigma u^{+}+(1-\sigma) u^{-}, x, t\right) S_{\eta}\left(\sigma u^{+}+(1-\sigma) u^{-}-v\right) d \sigma\right. \\
& \left.+\int_{0}^{1} \int_{\sigma u^{+}+(1-\sigma) u^{-}}^{v} a^{i j}(s) S_{\eta}\left(s-\sigma u^{+}-(1-\sigma) u^{-}\right) d \sigma d s\right] \frac{\partial u}{\partial x_{i}} \phi j_{h x_{j}} d x d t d y d \tau \\
& -\iint_{Q_{T}}^{u} \iint_{Q_{T}}^{u} \int_{v}^{i j} a_{x_{i}}^{i j}(\sigma, x, t) S_{\eta}(\sigma-v) \phi j_{h x_{j}} d \sigma d x d y d t d \tau \\
= & I_{31}+I_{32}-I_{7} .
\end{aligned}
$$

Also we notice that

$$
\begin{aligned}
& \sum_{i=1}^{N} \iint_{Q_{T}} \iint_{Q_{T}} S_{\eta}^{\prime}(u-v)\left(\left|g^{i}(u, x, t)\right|^{2}+\left|g^{i}(v, y, \tau)\right|^{2}\right) \psi d x d t d y d \tau \\
& \quad=\sum_{i=1}^{N} \iint_{Q_{T}} \iint_{Q_{T}} S_{\eta}^{\prime}(u-v)\left(\left|g^{i}(u, x, t)\right|-\left|g^{i}(v, y, \tau)\right|\right)^{2} \psi d x d t d y d \tau \\
& \quad+2 \sum_{i=1}^{N} \iint_{Q_{T}} \iint_{Q_{T}} S_{\eta}^{\prime}(u-v) g^{i}(u, x, t) g^{i}(v, y, \tau) \psi d x d t d y d \tau \\
& =I_{41}+I_{42} .
\end{aligned}
$$

We are able to prove that (see the details in the next section)

$$
\begin{aligned}
& \lim _{h \rightarrow 0} \lim _{\eta \rightarrow 0}\left(I_{32}+I_{42}\right) \\
& =2 \lim _{h \rightarrow 0} \iint_{Q_{T}} \iint_{Q_{T}} \gamma_{y_{j}}^{k j}(u, y, \tau) \operatorname{sgn}(v-u) \gamma^{i k}(u, x, t) u_{x_{i}} \phi j_{h} d x d t d y d \tau \\
& \quad=2 \iint_{Q_{T}} \iint_{Q_{T}} \gamma_{x_{j}}^{k j}(u, x, t) \operatorname{sgn}(v-u) \gamma^{i k}(u, x, t) u_{x_{i}} \phi d x d t,
\end{aligned}
$$

and clearly

$$
\begin{aligned}
I_{8} & =-\iint_{Q_{T}} \iint_{Q_{T}} \int_{u}^{v} a_{y_{j}}^{i j}(s, y, \tau) S_{\eta}(s-u) d s u_{x_{i}} \phi j_{h} d x d t d y d \tau \\
& =-\iint_{Q_{T}} \iint_{Q_{T}} \int_{u}^{v}\left[a_{y_{j}}^{i j}(s, y, \tau)-a_{y_{j}}^{i j}(u, y, \tau)\right] S_{\eta}(s-u) d s u_{x_{i}} \phi j_{h} d x d t d y d \tau
\end{aligned}
$$




$$
\begin{aligned}
& -\iint_{Q_{T}} \iint_{Q_{T}} \int_{u}^{v} a_{y_{j}}^{i j}(u, y, \tau) S_{\eta}(v-u) d s u_{x_{i}} \phi j_{h} d x d t d y d \tau \\
\rightarrow & -\iint_{Q_{T}} \iint_{Q_{T}} \int_{u}^{v} a_{y_{j}}^{i j}(u, y, \tau) \operatorname{sgn}(v-u) d s u_{x_{i}} \phi j_{h} d x d t d y d \tau \quad(\text { as } \eta \rightarrow 0) \\
= & -2 \iint_{Q_{T}} \iint_{Q_{T}} \int_{u}^{v} \gamma^{i k}(u, y, \tau) \gamma_{y_{j}}^{k j}(u, y, \tau) \operatorname{sgn}(v-u) d s u_{x_{i}} \phi j_{h} d x d t d y d \tau \\
\rightarrow & -2 \iint_{Q_{T}} \int_{u}^{v} \gamma^{i k}(u, x, t) \gamma_{x_{j}}^{k j}(u, x, t) \operatorname{sgn}(v-u) d s u_{x_{i}} \phi d x d t \quad(\text { as } h \rightarrow 0) \\
= & -\lim _{h \rightarrow 0} \lim _{\eta \rightarrow 0}\left(I_{32}+I_{42}\right) .
\end{aligned}
$$

Now, since

$$
\lim _{\eta \rightarrow 0} A_{\eta}^{i j}(u, x, t, v)=\lim _{\eta \rightarrow 0} A_{\eta}^{i j}(v, y, \tau, u)=\operatorname{sgn}(u-v)\left(A^{i j}(u, x, t)-A^{i j}(v, y, \tau)\right),
$$

we have

$$
\lim _{\eta \rightarrow 0}\left(A_{\eta}^{i j}(u, x, t, v) \phi_{x_{i}} j_{h x_{j}}-A_{\eta}^{i j}(u, y, \tau, v) \phi_{x_{i}} j_{h y_{j}}\right)=0 .
$$

At the same time,

$$
\begin{aligned}
& \lim _{\eta \rightarrow 0} \int_{v}^{u} b_{i x_{i}}(s, x, t) S_{\eta}^{\prime}(s-v) d s \\
& \quad=\lim _{\eta \rightarrow 0} \int_{v}^{u}\left[b_{i x_{i}}(s, x, t)-b_{i x_{i}}(v, x, t)\right] S_{\eta}^{\prime}(s-v) d s+b_{i x_{i}}(v, x, t) \operatorname{sgn}(u-v) \\
& \quad=b_{i x_{i}}(v, x, t) \operatorname{sgn}(u-v) .
\end{aligned}
$$

Likewise, we have

$$
\lim _{\eta \rightarrow 0} \int_{v}^{u} b_{i y_{i}}(s, x, t) S_{\eta}^{\prime}(s-u) d s=b_{i y_{i}}(v, x, t) \operatorname{sgn}(v-u)
$$

Combing (3.3)-(3.16), and letting $\eta \rightarrow 0, h \rightarrow 0$, we get

$$
\begin{aligned}
& \iint_{Q_{T}}\left\{|u(x, t)-v(x, t)| \phi_{t}-\operatorname{sgn}(u-v)\left(b_{i}(u, x, t)-b_{i}(v, x, t)\right) \phi_{x_{i}}\right. \\
& \quad+\operatorname{sgn}(u-v)\left(A^{i j}(u, x, t)-A^{i j}(v, x, t)\right) \phi_{x_{i} x_{j}}+\int_{v}^{u} a_{x_{j}}^{i j}(s, x, t) \operatorname{sgn}(s-v) d s \phi_{x_{i}} \\
& \quad+2 \int_{u}^{v} a_{x_{j}}^{i j}(s, x, t) \operatorname{sgn}(\tau-u) d \tau \phi_{x_{i}}-b_{i x_{i}}(v, x, t) \operatorname{sgn}(u-v) \phi \\
& \left.\quad-b_{i y_{i}}(u, x, t) \operatorname{sgn}(v-u) \phi\right\} d x d t \geq 0 .
\end{aligned}
$$

Let $\delta_{\varepsilon}$ be the mollifier as usual. If $y=\left(x_{1}, \ldots, x_{N}\right)$, then

$$
\delta(y)= \begin{cases}\frac{1}{A} e^{\frac{1}{|y|^{2}-1}}, & \text { if }|y|<1 \\ 0, & \text { if }|y| \geq 1\end{cases}
$$


where

$$
A=\int_{B_{1}(0)} e^{\frac{1}{|y|^{2}-1}} d x
$$

For any given $\varepsilon>0, \delta_{\varepsilon}(y)$ is defined as

$$
\delta_{\varepsilon}(y)=\frac{1}{\varepsilon^{N}} \delta\left(\frac{y}{\varepsilon}\right)
$$

Especially, we can choose $\phi$ in (3.17) by

$$
\phi(x, t)=\omega_{\lambda \varepsilon}(x) \eta(t)
$$

where $\eta(t) \in C_{0}^{\infty}(0, T), \omega_{\lambda \varepsilon}(x)$ is defined as follows. Let $\omega_{\lambda}(x) \in C_{0}^{2}(\Omega)$ be a function satisfying, for any given small enough $0<\lambda, 0 \leq \omega_{\lambda} \leq 1,\left.\omega\right|_{\partial \Omega}=0$, and

$$
\omega_{\lambda}(x)=1, \quad \text { if } d(x)=\operatorname{dist}(x, \partial \Omega) \geq \lambda,
$$

when $0 \leq d(x) \leq \lambda$,

$$
\omega_{\lambda}(d(x))=1-\frac{(d(x)-\lambda)^{2}}{\lambda^{2}} .
$$

Then we define

$$
\begin{aligned}
& \omega_{\lambda \varepsilon}=\omega_{\lambda} * \delta_{\varepsilon}(d), \\
& \omega_{\lambda \varepsilon}^{\prime}(d)=\int_{\{|s|<\varepsilon\} \cap\{0<d-s<\lambda\}} \omega_{\lambda}^{\prime}(d-s) \delta_{\varepsilon}(s) d s=-\int_{\{|s|<\varepsilon\} \cap\{0<d-s<\lambda\}} \frac{2(d-s-\lambda)}{\lambda^{2}} \delta_{\varepsilon}(s) d s .
\end{aligned}
$$

Clearly,

$$
\begin{aligned}
& \left|\omega_{\lambda \varepsilon}^{\prime}(d)\right| \leq \frac{c}{\lambda} . \\
& \omega_{\lambda \varepsilon}^{\prime \prime}(d)=-\frac{2}{\lambda^{2}} \int_{\{|s|<\varepsilon\} \cap\{0<d-s<\lambda\}} \delta_{\varepsilon}(s) d s .
\end{aligned}
$$

Now,

$$
\begin{aligned}
\phi_{x_{i} x_{j}} & =\eta(t)\left(\omega_{\lambda \varepsilon}(d(x))\right)_{x_{i} x_{j}}=\eta(t)\left(\omega_{\lambda \varepsilon}^{\prime}(d) d_{x_{i}}\right)_{x_{j}} \\
& =\eta(t)\left[\omega_{\lambda \varepsilon}^{\prime \prime}(d) d_{x_{i}} d_{x_{j}}+\omega_{\lambda \varepsilon}^{\prime}(d) d_{x_{i} x_{j}}\right] \\
& =\eta(t)\left[-\frac{2}{\lambda^{2}} d_{x_{i}} d_{x_{j}} \int_{\{|s|<\varepsilon\} \cap\{0<d-s<\lambda\}} \delta_{\varepsilon}(s) d s+\omega_{\lambda \varepsilon}^{\prime}(d) d_{x_{i} x_{j}}\right],
\end{aligned}
$$

using the conditions $\left|d_{x_{i} x_{j}}\right| \leq c$, and using the fact of that $|\nabla d|=1$, noticing that

$$
\operatorname{sgn}(u-v)\left(A^{i j}(u, x, t)-A^{i j}(v, x, t)\right) d_{x_{i}} d_{x_{j}}=|u-v| a^{i j}(\zeta, x, t) d_{x_{i}} d_{x_{j}} \geq 0,
$$


where $\zeta \in(v, u)$. Then by (1.20), from (3.15), we have

$$
\iint_{Q_{T}}|u(x, t)-v(x, t)| \phi_{t} d x d t+c \int_{0}^{T} \int_{\Omega_{\lambda}} \eta(t)\left|\omega_{\lambda \varepsilon}^{\prime}(d)\right||u-v| d x d t \geq 0
$$

where $\Omega_{\lambda}=\{x \in \Omega: d(x, \partial \Omega)<\lambda\}$.

According to the definition of the trace (2.3), let $\lambda \rightarrow 0$ in (3.21). By (3.18)-(3.21), we have

$$
c \operatorname{essup}_{\Sigma_{2} \times(0, T)}|f(x, t)-g(x, t)|+\iint_{Q_{T}}|u(x, t)-v(x, t)| \eta_{t}^{\prime} d x d t \geq 0 .
$$

Let $0<\tau<s<T$, and

$$
\eta(t)=\int_{\tau-t}^{s-t} \alpha_{\varepsilon}(\sigma) d \sigma, \quad \varepsilon<\min \{\tau, T-s\} .
$$

Here $\alpha_{\varepsilon}(t)$ is the kernel of the mollifier with $\alpha_{\varepsilon}(t)=0$ for $t \notin(-\varepsilon, \varepsilon)$. Then

$$
c \operatorname{essup}_{\Sigma_{2} \times(0, T)}|f(x, t)-g(x, t)|+\int_{0}^{T}\left[\alpha_{\varepsilon}(\tau-t)-\alpha_{\varepsilon}(s-t)\right]|u-v|_{L^{1}(\Omega)} d t \geq 0 .
$$

Let $\varepsilon \rightarrow 0$. Then

$$
|u(x, s)-v(x, s)|_{L^{1}(\Omega)} \leq|u(x, \tau)-v(x, \tau)|_{L^{1}(\Omega)}+c \operatorname{essup}_{\Sigma_{2} \times(0, T)}|f(x, t)-g(x, t)|
$$

and the desired result follows by letting $\tau \rightarrow 0$.

\section{The proof of (3.12)}

To prove (3.12), we have to make some basic calculations. By the properties of the BV functions (Lemma 3.1 and Lemma 3.7.8 of [2]),

$$
\begin{aligned}
& \int_{\Gamma^{u}} \int_{\Gamma^{v}} \partial_{y_{j}} \partial_{x_{i}} \int_{v}^{u} \gamma^{i k}(\delta, x, t) \int_{\delta}^{v} \gamma^{k j}(\sigma, y, \tau) S_{\eta}^{\prime}(\sigma-\delta) d \delta d \sigma \psi \\
& \quad=\int_{\Gamma^{u}} \int_{\Gamma^{v}} \partial_{y_{j}} \int_{u^{-}}^{u^{+}} \gamma^{i k}(\delta, x, t) \int_{\delta}^{v} \gamma^{k j}(\sigma, y, \tau) S_{\eta}^{\prime}(\sigma-\delta) d \delta d \sigma \psi v_{i} d H_{x}=0,
\end{aligned}
$$

and likewise

$$
\begin{aligned}
& \int_{Q_{T} \backslash \Gamma^{u}} \int_{\Gamma^{v}} \partial_{y_{j}} \partial_{x_{i}} \int_{v}^{u} \gamma^{i k}(\delta, x, t) \int_{\delta}^{v} \gamma^{k j}(\sigma, y, \tau) S_{\eta}^{\prime}(\sigma-\delta) d \delta d \sigma \psi=0, \\
& \int_{\Gamma^{u}} \int_{Q_{T} \backslash \Gamma^{v}} \partial_{y_{j}} \partial_{x_{i}} \int_{v}^{u} \gamma^{i k}(\delta, x, t) \int_{\delta}^{v} \gamma^{k j}(\sigma, y, \tau) S_{\eta}^{\prime}(\sigma-\delta) d \delta d \sigma \psi=0 .
\end{aligned}
$$

So

$$
\begin{aligned}
& \iint_{Q_{T}} \iint_{Q_{T}} \partial_{x_{i}} \partial_{y_{j}} \int_{v}^{u} \gamma^{i k}(\delta, x, t) \int_{\delta}^{v} \gamma^{k j}(\sigma, y, \tau) S_{\eta}^{\prime}(\sigma-\delta) d \sigma d \delta \psi d x d t d y d \tau \\
& \quad=\iint_{Q_{T}} \iint_{Q_{T}}\left[\psi \partial_{y_{j}} \int_{0}^{1} \gamma^{n i}\left(s u^{+}-(1-s) u^{-}, x, t\right)\right.
\end{aligned}
$$




$$
\begin{aligned}
& \cdot \int_{s u^{+}+(1-s) u^{-}}^{v} \gamma^{k j}(\sigma, y, \tau) S_{\eta}^{\prime}\left(\sigma-s u^{+}-(1-s) u^{-}\right) u_{x_{i}} d \sigma d s \\
& \left.+\psi \partial_{y_{j}} \int_{v}^{u} \gamma_{x_{i}}^{k i}(\delta, x, t) \int_{\delta}^{v} \gamma^{k j}(\sigma, y, \tau) S_{\eta}^{\prime}(\sigma-\delta) d \delta d \sigma\right] d x d t d y d \tau \\
= & \iint_{Q_{T} \backslash \Gamma^{u}} \iint_{Q_{T} \backslash \Gamma^{v}}\left[\psi \partial_{y_{j}} \int_{0}^{1} \gamma^{n i}\left(s u^{+}-(1-s) u^{-}, x, t\right)\right. \\
& \cdot \int_{s u^{+}+(1-s) u^{-}}^{v} \gamma^{k j}(\sigma, y, \tau) S_{\eta}^{\prime}\left(\sigma-s u^{+}-(1-s) u^{-}\right) u_{x_{i}} d \sigma d s \\
& \left.+\psi \partial_{y_{j}} \int_{v}^{u} \gamma_{x_{i}}^{k i}(\delta, x, t) \int_{\delta}^{v} \gamma^{k j}(\sigma, y, \tau) S_{\eta}^{\prime}(\sigma-\delta) d \delta d \sigma\right] d x d t d y d \tau \\
= & J_{1-5}+J_{6} .
\end{aligned}
$$

Since in $Q_{T} \backslash \Gamma_{u}, u^{+}=u^{-}$, in $Q_{T} \backslash \Gamma_{v}, v^{+}=v^{-}$, we can deal with $J_{1-5}$ as

$$
\begin{aligned}
& \partial_{y_{j}} \int_{0}^{1} \gamma^{i k}\left(s u^{+}+(1-s) u^{-}\right) \int_{s u^{+}+(1-s) u^{-}}^{v} \gamma^{k j}(\sigma) S_{\eta}^{\prime}\left(\sigma-s u^{+}-(1-s) u^{-}\right) u_{x_{i}} d \sigma d s \\
& =\gamma^{i k}(u, x, t)\left[\gamma^{k j}(\nu, y, \tau) S_{\eta}^{\prime}(\nu-u) u_{x_{i}} v_{y_{j}}+\int_{u}^{v} \gamma_{y_{j}}^{k j}(\sigma, y, \tau) S_{\eta}^{\prime}(\sigma-u) u_{x_{i}} d \sigma\right] \\
& =\partial_{x_{i}} \int_{v}^{u} \gamma^{i k}(\delta, x, t) d \delta-\int_{v}^{u} \gamma_{x_{i}}^{i k}(\delta, x, t) d \delta\left[\partial_{y_{j}} \int_{u}^{v} \gamma^{k j}(\sigma, y, \tau) d \sigma\right. \\
& \left.-\int_{u}^{v} \gamma_{y_{j}}^{k j}(\sigma, y, \tau) d \sigma\right] S_{\eta}^{\prime}(\nu-u)+\gamma^{i k}(u, x, t) u_{x_{i}} \int_{u}^{v} \gamma_{y_{j}}^{k j}(\sigma, y, \tau) S_{\eta}^{\prime}(\sigma-u) d \sigma \\
& =\partial_{x_{i}} \int_{v}^{u} \gamma^{i k}(\delta, x, t) d \delta \partial_{y_{j}} \int_{u}^{v} \gamma^{k j}(\sigma, y, \tau) d \sigma S_{\eta}^{\prime}(v-u) \\
& -\partial_{x_{i}} \int_{v}^{u} \gamma^{i k}(\delta, x, t) d \delta \int_{u}^{v} \gamma_{y_{j}}^{k j}(\sigma, y, \tau) d \sigma S_{\eta}^{\prime}(v-u) \\
& -\int_{v}^{u} \gamma_{x_{i}}^{i k}(\delta, x, t) d \delta \partial_{y_{j}} \int_{u}^{v} \gamma^{k j}(\sigma, y, \tau) d \sigma S_{\eta}^{\prime}(v-u) \\
& +\int_{v}^{u} \gamma_{x_{i}}^{i k}(\delta, x, t) d \delta \int_{u}^{v} \gamma_{y_{j}}^{k j}(\sigma, y, \tau) d \sigma S_{\eta}^{\prime}(v-u) \\
& +\gamma^{i k}(u, x, t) u_{x_{i}} \int_{u}^{v} \gamma_{y_{j}}^{k j}(\sigma, y, \tau) S_{\eta}^{\prime}(\sigma-u) d \sigma \\
& =j_{1}+j_{2}+j_{3}+j_{4}+j_{5} \text {, } \\
& J_{1-5}=\iint_{Q_{T}}\left(j_{1}+j_{2}+j_{3}+j_{4}+j_{5}\right) d x d t d y d \tau=J_{1}+J_{2}+J_{3}+J_{4}+J_{5} \text {. }
\end{aligned}
$$

By (4.1),

$$
\begin{aligned}
& \iint_{Q_{T}} \iint_{Q_{T}} \partial_{x_{i}} \partial_{y_{j}} \int_{v}^{u} \gamma^{i k}(\delta, x, t) \int_{\delta}^{v} \gamma^{k j}(\sigma, y, \tau) S_{\eta}^{\prime}(\sigma-\delta) d \sigma d \delta \psi d x d t d y d \tau \\
& \quad=J_{1}+J_{2}+J_{3}+J_{4}+J_{5}+J_{6} .
\end{aligned}
$$


At the same time,

$$
\begin{aligned}
\iint_{Q_{T}} & \iint_{Q_{T}} \partial_{x_{i}} \partial_{y_{j}} \int_{v}^{u} \gamma^{i k}(\delta, x, t) \int_{\delta}^{v} \gamma^{k j}(\sigma, y, \tau) S_{\eta}^{\prime}(\sigma-\delta) d \sigma d \delta \psi d x d t d y d \tau \\
= & \iint_{Q_{T}} \iint_{Q_{T}}\left[\psi \partial_{y_{j}} \int_{0}^{1} \gamma^{i k}\left(s u^{+}+(1-s) u^{-}, x, t\right)\right. \\
& \cdot \int_{s u^{+}+(1-s) u^{-}}^{v} \gamma^{k j}(\sigma, y, \tau) S_{\eta}^{\prime}\left(\sigma-s u^{+}-(1-s) u^{-}\right) u_{x_{i}} d \sigma d s \\
& \left.+\psi \partial_{y_{j}} \int_{v}^{u} \gamma_{x_{i}}^{i k}(\delta, x, t) \int_{\delta}^{v} \gamma^{k j}(\sigma, y, \tau) S_{\eta}^{\prime}(\sigma-\delta) d \sigma d \delta\right] d x d t d y d \tau \\
= & \iint_{Q_{T}} \iint_{Q_{T}}\left[\phi j_{h x_{j}} \int_{0}^{1} \gamma^{i k}\left(s u^{+}+(1-s) u^{-}, x, t\right)\right. \\
& \cdot \int_{s u^{+}+(1-s) u^{-}}^{v} \gamma^{k j}(\sigma, y, \tau) S_{\eta}^{\prime}\left(\sigma-s_{1} u^{+}-\left(1-s_{1}\right) u^{-}\right) u_{x_{i}} d \sigma d s \\
& +\phi j_{h} \int_{v}^{u} \gamma_{x_{i}}^{i k}(\delta, x, t)\left[\int_{0}^{1} \gamma^{k j}\left(s v^{+}+(1-s) v^{-}, y, \tau\right) S_{\eta}^{\prime}\left(s v^{+}+(1-s) v^{-}-\delta\right) v_{y_{j}} d s\right. \\
& \left.\left.+\int_{\delta}^{v} \gamma_{y_{j}}^{k j}(\sigma, y, \tau) S_{\eta}^{\prime}(\sigma-\delta) d \sigma\right]\right] d \delta d x d t d y d \tau=J_{6}+J_{7} \cdot
\end{aligned}
$$

Comparing (4.2) to (4.3), one has

$$
J_{1}=J_{7}-\left(J_{2}+J_{3}+J_{4}+J_{5}\right) .
$$

So

$$
\begin{aligned}
I_{32}+ & I_{42} \\
= & \iint_{Q_{T}} \iint_{Q_{T}}\left\{\int_{0}^{1} a^{i j}\left(s u^{+}+(1-s) u^{-}, x, t\right) S_{\eta}\left(s u^{+}+(1-s) u^{-}-v\right) d s\right. \\
& \left.-\int_{0}^{1} \int_{s u^{+}+(1-s) u^{-}}^{v} a^{i j}(\sigma, y, \tau) S_{\eta}^{\prime}\left(\sigma-s u^{+}-(1-s) u^{-}\right) d s d \sigma\right\} d x d t d y d \tau \\
& +2 \iint_{Q_{T}} \iint_{Q_{T}} S_{\eta}^{\prime}(u-v) \partial_{x_{i}} \int_{v}^{u} \gamma^{i k}(\delta, x, t) d \delta \cdot \partial_{y_{j}} \int_{u}^{v} \gamma^{k j}(\sigma, y, \tau) d \sigma \psi d x d t d y d \tau \\
= & \iint_{Q_{T}} \iint_{Q_{T}}\left\{\int_{0}^{1} a^{i j}\left(s u^{+}+(1-s) u^{-}, x, t\right) S_{\eta}\left(s u^{+}+(1-s) u^{-}-v\right) d s\right. \\
& \left.-\int_{0}^{1} \int_{s u^{+}+(1-s) u^{-}}^{v} a^{i j}(\sigma, y, \tau) S_{\eta}^{\prime}\left(\sigma-s u^{+}-(1-s) u^{-}\right) d s d \sigma\right\} d x d t d y d \tau+2 J_{1} . \quad(4.5)
\end{aligned}
$$

Substituting (4.4) into (4.5), we have

$$
\begin{aligned}
I_{32}+ & I_{42} \\
= & \iint_{Q_{T}} \iint_{Q_{T}}\left\{-\int_{0}^{1} \int_{s u^{+}+(1-s) u^{-}}^{v} \gamma^{i k}\left(s u^{+}+(1-s) u^{-}, x, t\right) \gamma^{k j}\left(s u^{+}+(1-s) u^{-}, x, t\right)\right. \\
& \cdot S_{\eta}^{\prime}\left(\sigma-s u^{+}-(1-s) u^{-}\right) d \sigma d s
\end{aligned}
$$




$$
\begin{aligned}
& -\int_{0}^{1} \int_{s u^{+}+(1-s) u^{-}}^{v} \gamma^{i k}(\sigma, y, \tau) \gamma^{k j}(\sigma, y, \tau) S_{\eta}^{\prime}\left(\sigma-s u^{+}-(1-s) u^{-}\right) d \sigma d s \\
& +2 \int_{0}^{1} \gamma^{i k}\left(s u^{+}+(1-s) u^{-}, x, t\right) \int_{s u^{+}+(1-s) u^{-}}^{v} \gamma^{i k}(\sigma, y, \tau) \\
& \left.+S_{\eta}^{\prime}\left(\sigma-s u^{+}-(1-s) u^{-}\right) d \sigma d s\right\} u_{x_{i}} j_{h x_{j}} \phi d x d t d y d \tau \\
& +2 \iint_{Q_{T}}\left\{\iint_{Q_{T}} \partial_{x_{i}} \int_{v}^{u} \gamma^{i k}(\delta, x, t) d \delta \int_{u}^{v} \gamma_{y_{j}}^{k j}(\sigma, y, \tau) d \sigma S_{\eta}^{\prime}(v-u)\right. \\
& -S_{\eta}^{\prime}(v-u)\left[\int_{v}^{u} \gamma_{x_{i}}^{i k}(\delta, x, t) d \delta \cdot \partial_{y_{j}} \int_{u}^{v} \gamma^{k j}(\sigma, y, \tau) d \sigma\right. \\
& \left.-\int_{v}^{u} \gamma_{x_{i}}^{i k}(\delta, x, t) d \delta \int_{u}^{v} \gamma_{y_{j}}^{k j}(\sigma, y, \tau) d \sigma\right] \\
& -\gamma^{i k}(u, x, t) u_{x_{i}} \int_{u}^{v}\left[\gamma_{y_{j}}^{k j}(\sigma, y, \tau)-\gamma_{y_{j}}^{k j}(u, y, \tau)\right] S_{\eta}^{\prime}(\sigma-u) d \sigma \\
& \left.+\gamma^{i k}(u, x, t) u_{x_{i}} \gamma_{y_{j}}^{k j}(u, y, \tau) S_{\eta}(v-u)\right\} \phi j_{h} d x d t d y d \tau .
\end{aligned}
$$

We have

$$
\begin{aligned}
\gamma^{i k}\left(s u^{+}+(1-s) u^{-}, x, t\right)\left[\gamma^{i k}(\sigma, y, \tau)-\gamma^{k j}\left(s u^{+}+(1-s) u^{-}, x, t\right)\right] \\
\cdot S_{\eta}^{\prime}\left(\sigma-s u^{+}-(1-s) u^{-}\right) j_{h x_{j}} \\
+\gamma^{k j}(\sigma, y, \tau)\left[\gamma^{i k}\left(s u^{+}+(1-s) u^{-}, x, t\right)-\gamma^{i k}(\sigma, y, \tau)\right] S_{\eta}^{\prime}\left(\sigma-s u^{+}-(1-s) u^{-}\right) j_{h x_{j}} \\
=\left[\gamma^{i k}\left(s u^{+}+(1-s) u^{-}, x, t\right)-\gamma^{i k}(\sigma, x, t)\right] S_{\eta}^{\prime}\left(\sigma-s u^{+}-(1-s) u^{-}\right) \\
\cdot\left[\gamma^{k j}(\sigma, y, \tau)-\gamma^{k j}\left(s u^{+}+(1-s) u^{-}, x, t\right)\right] j_{h x_{j}} \\
+\left[\gamma^{k j}(\sigma, y, \tau)-\gamma^{k j}\left(s u^{+}+(1-s) u^{-}, y, \tau\right)\right] S_{\eta}^{\prime}\left(\sigma-s u^{+}-(1-s) u^{-}\right) \\
\cdot\left[\gamma^{k j}\left(s u^{+}+(1-s) u^{-}, x, t\right)-\gamma^{k j}(\sigma, y, \tau)\right] j_{h x_{j}} \\
+\left[\gamma^{k j}(\sigma, y, \tau)-\gamma^{k j}\left(s u^{+}+(1-s) u^{-}, x, t\right)\right] \\
\cdot\left[\gamma^{i k}(\sigma, x, t)-\gamma^{i k}\left(s u^{+}+(1-s) u^{-}, y, \tau\right)\right] j_{h x_{j}} \\
\cdot S_{\eta}^{\prime}\left(\sigma-s u^{+}-(1-s) u^{-}\right) .
\end{aligned}
$$

Now, by $\lim _{\eta \rightarrow 0} s S_{\eta}^{\prime}(s)=0$, by (1.20), and $\lim _{h \rightarrow 0} \omega_{h}^{\prime}(s) s^{2+\delta}=0$, as $\eta \rightarrow 0, h \rightarrow 0$, every term of the right-hand side of (4.6) approaches 0 except the last term. The last term approaches

$$
2 \iint_{Q_{T}} \iint_{Q_{T}} \gamma_{x_{j}}^{k j}(u, x, t) \operatorname{sgn}(v-u) \gamma^{i k}(u, x, t) u_{x_{i}} \phi d x d t
$$

so we have (3.12).

\section{Proof of Lemma 3.1}

Let $u$ be a solution of equation (1.1) in the sense of Definition 1.1, we want to prove

$$
\int_{u^{-}}^{u^{+}} \gamma^{i j}(s, x, t) d s \cdot v_{i}=0, \quad \text { a.e. }(x, t) \text { on } \Gamma_{u}, j=1,2, \ldots, N \text {. }
$$


Denote

$$
\begin{aligned}
& \Gamma_{1}=\left\{(x, t) \in \Gamma_{u}, v_{1}(x, t)=\cdots=v_{N}(x, t)=0\right\}, \\
& \Gamma_{2}=\left\{(x, t) \in \Gamma_{u}, v_{1}^{2}(x, t)+\cdots+v_{N}^{2}(x, t)>0\right\} .
\end{aligned}
$$

First we prove $H\left(\Gamma_{1}\right)=0$. Since any measurable subset of $\Gamma_{1}$ can be expressed as the union of Borel sets and a set of measure zero, it suffices to prove

$$
H(U)=0,
$$

where $U$ is a Borel subset of $\Gamma_{1}$. We may suppose $\bar{U}$ is compact. By Lemma 3.7.8 in [2], for any bounded function $f(x, t)$, which is measurable with respect to the measure $\frac{\partial u}{\partial x_{i}}$, we have

$$
\iint_{U} f(x, t) \frac{\partial u}{\partial x_{i}}=\int_{0}^{T} d t \int_{U^{t}} f(x, t) \frac{\partial u}{\partial x_{i}}
$$

where $U^{t}=\{x:(x, t) \in U\}$. By [15], for any Borel subset $S \subset U, S^{t} \subset U^{t}$, for $i=1,2, \ldots, N$,

$$
\begin{aligned}
& \frac{\partial u}{\partial x_{i}}(S)=\int_{S}\left(u^{+}(x, t)-u^{-}(x, t)\right) v_{i} d H, \\
& \frac{\partial u(\cdot, t)}{\partial x_{i}}\left(S^{t}\right)=\int_{S^{t}}\left(u_{+}^{t}(x, t)-u_{-}^{t}(x, t)\right) v_{i} d H^{t}
\end{aligned}
$$

Equation (5.2) is equivalent to

$$
\begin{aligned}
& \iint_{U} f(x, t)\left(u^{+}(x, t)-u^{-}(x, t)\right) v_{i} d H \\
& =\int_{0}^{T} d t \int_{U^{t}} f(x, t)\left(u_{+}^{t}(x, t)-u_{-}^{t}(x, t)\right) v_{i}^{t} d H^{t} .
\end{aligned}
$$

The definition of $\Gamma_{1}$ implies that the left-hand side vanishes, so we have

$$
\int_{0}^{T} d t \int_{U^{t}} f(x, t)\left(u_{+}^{t}(x, t)-u_{-}^{t}(x, t)\right) v_{i}^{t} d H^{t}=0 .
$$

Choose $f(x, t)=\chi_{u}(x, t) \operatorname{sgn}\left(u_{+}^{t}(x, t)-u_{-}^{t}(x, t)\right) \operatorname{sgn} v_{i}^{t}$, where $\chi_{u}(x, t)$ denotes the characteristic function of $U$, sum up for $i$ from 1 up to $N$. Then we obtain

$$
\int_{G} d t \int_{U^{t}}\left(u_{+}^{t}(x, t)-u_{-}^{t}(x, t)\right)\left(\left|v_{1}^{t}\right|+\cdots+\left|v_{N}^{t}\right|\right) d H^{t}=0
$$

where $G$ is the projection of $U$ on the $t$-axis. Equation (5.3) implies, for almost all $t \in G$,

$$
\int_{U^{t}}\left(u_{+}^{t}(x, t)-u_{-}^{t}(x, t)\right)\left(\left|v_{1}^{t}\right|+\cdots+\left|v_{N}^{t}\right|\right) d H^{t}=0
$$

and hence, for almost all $t \in G$,

$$
v_{1}^{t}=\cdots=v_{N}^{t}=0
$$


$H^{t}$-almost everywhere on $U^{t}$, which is impossible unless mes $G=0$.

For any $\alpha, \beta$ with $0<\alpha<\beta<T$, we choose $\psi_{l}(t) \in C_{0}^{\infty}(0, T)$ such that

$$
0 \leq \psi_{l}(t) \leq 1, \quad \lim _{l \rightarrow \infty} \psi_{l}(t)=\chi_{[\alpha, \beta]}(t), \quad \forall t \in[0, T]
$$

By [2], we can choose $\varphi_{n} \in C_{0}^{\infty}\left(Q_{T}\right)$ such that

$$
\left|\varphi_{n}(x, t)\right| \leq 1, \quad \lim _{n \rightarrow \infty} \varphi_{n}=\chi_{U} \quad \text { in } L^{1}\left(Q_{T},\left|\frac{\partial u}{\partial t}\right|\right) .
$$

Now, recalling that $A^{i j}(u, x, t)=\int_{0}^{u} a^{i j}(s, x, t) d s$, for any $\phi \in C_{0}^{\infty}\left(Q_{T}\right)$,

$$
\begin{aligned}
& \iint_{Q_{T}} \frac{\partial}{\partial x_{i}} a^{i j}(u, x, t) \frac{\partial u}{\partial x_{j}} \phi(x, t) d x d t \\
& \quad=-\iint_{Q_{T}} a^{i j}(u, x, t) \frac{\partial u}{\partial x_{j}} \phi_{x_{i}} d x d t \\
& \quad=-\iint_{Q_{T}}\left[\frac{\partial A^{i j}(u, x, t)}{\partial x_{i}}-\int_{0}^{u} a_{x_{i}}^{i j}(s, x, t) d s \phi_{x_{j}}\right] d x d t \\
& \quad=\iint_{Q_{T}}\left[A^{i j}(u, x, t) \phi_{x_{i} x_{j}}-\int_{0}^{u} a_{x_{i}}^{i j}(s, x, t) d s \phi_{x_{j}}\right] d x d t, \\
& \iint \frac{\partial b_{i}(u, x, t)}{\partial x_{i}} \phi d x d t=-\iint_{Q_{T}} b_{i}(u, x, t) \phi_{x_{i}} d x d t .
\end{aligned}
$$

Let $\phi=\varphi_{n}(x, t) \psi_{l}(t)$. From the definition of the BV function, we have

$$
\begin{aligned}
& \iint_{Q_{T}} \varphi_{n}(x, t) \psi_{l}(t) \frac{\partial u}{\partial t} \\
& =-\iint_{Q_{T}} b_{i}(u, x, t) \frac{\partial}{\partial x_{i}} \varphi_{n}(x, t) \psi_{l}(t) d x d t \\
& \quad+\iint_{Q_{T}}\left[A^{i j}(u, x, t) \varphi_{n_{x_{i} x_{j}}}(x, t) \psi_{l}(t)-\int_{0}^{u} a_{x_{i}}^{i j}(s, x, t) d s \varphi_{n x_{j}}(x, t) \psi_{l}(t)\right] d x d t .
\end{aligned}
$$

Let $l \rightarrow \infty$. Then

$$
\begin{aligned}
& \iint_{Q_{T}} \varphi_{n}(x, t) \chi_{[\alpha, \beta]}(t) \frac{\partial u}{\partial t} \\
& =-\iint_{Q_{T}} b_{i}(u, x, t) \frac{\partial}{\partial x_{i}} \varphi_{n}(x, t) \chi_{[\alpha, \beta]}(t) d x d t \\
& \quad+\iint_{Q_{T}}\left[A^{i j}(u, x, t) \varphi_{n_{x_{i} x_{j}}}(x, t) \chi_{[\alpha, \beta]}(t)-\int_{0}^{u} a_{x_{i}}^{i j}(s, x, t) d s \varphi_{n x_{j}}(x, t) \chi_{[\alpha, \beta]}(t)\right] d x d t .
\end{aligned}
$$

Clearly, this equality also holds if $[\alpha, \beta]$ is replaced by $(\alpha, \beta)$ and hence it holds even if $[\alpha, \beta]$ is replaced by any open set $I$ with $\bar{I} \subset(0, T)$. Since $G$ is a Borel set, by approximation 
we may conclude that

$$
\begin{aligned}
& \iint_{Q_{T}} \varphi_{n}(x, t) \chi_{G}(t) \frac{\partial u}{\partial t} \\
& \quad=-\iint_{Q_{T}} b_{i}(u, x, t) \frac{\partial}{\partial x_{i}} \varphi_{n}(x, t) \chi_{G}(t) d x d t \\
& \quad+\iint_{Q_{T}}\left[A^{i j}(u, x, t) \varphi_{n_{x_{i} x_{j}}}(x, t) \chi_{G}(t)-\int_{0}^{u} a_{x_{i}}^{i j}(s, x, t) d s \varphi_{n x_{j}}(x, t) \chi_{G}(t)\right] d x d t .
\end{aligned}
$$

Since mes $G=0$, the three terms on the right-hand vanish and

$$
\iint_{Q_{T}} \varphi_{n}(x, t) \chi_{G}(t) \frac{\partial u}{\partial t}=0 .
$$

Let $n \rightarrow \infty$. Then

$$
\iint_{U} \frac{\partial u}{\partial t}=\iint_{Q_{T}} \chi_{U}(x, t) \chi_{G} \frac{\partial u}{\partial t}=0 .
$$

Hence

$$
\int_{U}\left(u^{+}(x, t)-u^{-}(x, t)\right) v_{t} d H=0
$$

which implies $H(U)=0$ and $H\left(\Gamma_{1}\right)=0$ by the arbitrariness of $U$.

Next, we prove that (5.1) is true in $\Gamma_{2}$. Let $U$ be any Borel subset of $\Gamma_{2}$ which is compact in $Q_{T}$. Since $U$ is a set of $N+1$-dimensional measure zero and $\frac{\partial}{\partial x_{i}} A^{i j}(u, x, t) \in L_{l o c}^{2}\left(Q_{T}\right)$, we have

$$
\iint_{U} \frac{\partial}{\partial x_{i}} A^{i j}(u, x, t) d x d t=0, \quad i=1, \ldots, N,
$$

and hence

$$
\int_{U}\left(A^{i j}\left(u^{+}(x, t)\right)-A^{i j}\left(u^{-}(x, t)\right)\right) v_{i} d H=0, \quad i=1, \ldots, N .
$$

By the arbitrariness of $U$, it follows by the definition of $\Gamma_{2}$ that

$$
\int_{u^{-}}^{u^{+}} a^{i j}(s, x, t) d x \cdot v_{i}=0, \quad \text { a.e. on } \Gamma_{2},
$$

then

$$
\int_{u^{-}}^{u^{+}} a^{i j}(s, x, t) d x v_{i} v_{j}=0, \quad \text { a.e. on } \Gamma_{2} .
$$

From this fact,

$$
\int_{u^{-}(x, t)}^{u^{+}(x, t)} \gamma^{i j}(s, x, t) d s \cdot v_{i}=0, \quad \text { a.e. on } \Gamma_{2} .
$$

Thus the lemma is proved. 


\section{Appendix 1: The boundary condition on Definition 1.1}

Clearly, if (1.2) and (1.6) are both true, equation (1.1) is not only degenerate in the interior of $\Omega$, but also degenerate on the boundary $\Sigma=\partial \Omega$ of $\Omega$. If equation (1.1) is weakly degenerate, we can impose the boundary condition (1.6) usually, one may refer to [2, 15-17, 23, 24 ]. But if equation (1.1) is strongly degenerate, it even is allowed to be completely degenerate, global solutions are in general discontinuous, the boundary condition (1.6) is not necessarily satisfied in the classical sense that a trace of the solution exists and equals the homogeneous value on $\Sigma$. Now, we give a very brief reviewing of the international way of how to deal with this problem.

In the completely degenerate case, equation (1.1) becomes a first order hyperbolic equation and it is well known that a smooth solution of equation (1.1) is constant along the maximal segment of the characteristic line in $Q_{T}$. Now suppose that this segment intersects both $\{0\} \times \Omega$ and $\Sigma$. Then the problem (1.1)-(1.5)-(1.6) would be overdetermined if (1.6) was assumed in the classical sense. Thus one needs to work within a suitable framework of entropy solutions and entropy boundary conditions to obtain uniqueness and existence results. In the BV setting, Bardos et al. [25] first gave an interpretation of the boundary condition (1.6) as an 'entropy' inequality on $\Sigma$, which is the so-called BLN condition. However, since the trace of solutions is involved in the formulation of the BLN condition, it makes no sense if the solution is merely in $L^{\infty}$. Otto [26] extended the Dirichlet problem for hyperbolic equations to the $L^{\infty}$ setting and proved a unique entropy solution by introducing an integral formulation of the boundary condition.

For degenerate parabolic equations, the isotropic diffusion case, $\left(a^{i j}\right)=a(u) I$, first had been developed around 2000. Besides the works in [8-11, 13], Carrillo [27] succeeded in proving the uniqueness and existence of entropy solutions under the homogeneous boundary condition, later Mascia et al. [12] and Michel and Vovelle [28] extended those results to the case of a nonhomogeneous boundary condition. At the same time, Kobayasi [29] proved the uniqueness by using the kinetic formulation introduced in [30, 31]. The initial-boundary value problem of the anisotropic case is more delicate and has been treated in more recent years. Bendahmane and Karlsen treated in [32] (also see [33, 34]) a class of doubly nonlinear degenerate parabolic equations with homogeneous Dirichlet boundary conditions. Kobayashi and Ohwa treated in [6] the general anisotropic case with nonhomogeneous boundary condition in the unit $\mathrm{N}$-dimensional cube, while, $\mathrm{Li}$ and Wang treat in [7] the isotropic case with homogeneous boundary condition in a general bounded domain. In other words, in all these works, in the international way, the boundary condition is not directly shown as (1.6) but is elegantly implicity contained in family entropy inequalities (for example, [6]), or it is treated in a special weak sense such as [7]. The most characteristic feature lies in that the boundary condition can be treated in the $L^{\infty}$ setting, and the uniqueness of the entropy solutions can be obtained. So, if we consider the Cauchy problem of equation (1.1), the international way has great superiority.

Unlike the international way, the Chinese way still treats the boundary condition in the classical sense, so it requires that the solution is regular at least in the BV sense. If the solution is only in the $L^{\infty}$ setting, it cannot be treated in the Chinese way. Certainly, as we have said before, the Chinese way has the advantages that it clearly shows the condition (1.6) generally is overdetermined, and only a portion of the boundary should be given the boundary value as we have shown in Theorem 1.2. In the following, we will give an explanation of the reasonableness of homogeneous value condition (1.13). 
In the 1950-1960s, Fichera [35, 36] and Oleinnik [37, 38] developed and perfected the general theory of second order equations with nonnegative characteristic form, which, in particular contain those degenerating on the boundary. We can call it Fichera-Oleinnik theory. By the theory, for a linear degenerate elliptic equation,

$$
\sum_{r, s=1}^{N+1} a^{r s}(x) \frac{\partial^{2} u}{\partial x_{r} \partial x_{s}}+\sum_{r=1}^{N+1} b_{r}(x) \frac{\partial u}{\partial x_{r}}+c(x) u=f(x), \quad x \in \widetilde{\Omega} \subset \mathbb{R}^{N+1}
$$

if one wants to consider the Dirichlet boundary value problem of equation (A.1), one only needs to give a partial boundary condition. In detail, let $\left\{n_{s}\right\}$ be the unit inner normal vector of $\partial \widetilde{\Omega}$ and denote

$$
\begin{aligned}
& \Sigma_{2}=\left\{x \in \partial \widetilde{\Omega}: a^{r s} n_{r} n_{s}=0,\left(b_{r}-a_{x_{s}}^{r s}\right) n_{r}<0\right\}, \\
& \Sigma_{3}=\left\{x \in \partial \widetilde{\Omega}: a^{r s} n_{s} n_{r}>0\right\} .
\end{aligned}
$$

Then, to ensure the well-posedness of equation (A.1), Fichera-Oleǐnik theory tells us that the suitable boundary condition is

$$
\left.u\right|_{\Sigma_{2} \cup \Sigma_{3}}=g(x)
$$

In particular, if the matrix $\left(a^{r s}\right)$ is positive definite, (A.2) is just the usual Dirichlet boundary condition.

Now, for the porous medium equation (1.3), or the general reaction-diffusion equation

$$
u_{t}=\Delta A(u)
$$

with the existence of $A^{-1}$, in other words, equation (A.3) is weakly degenerate, then let $v=A(u), u=A^{-1}(v)$. We have

$$
\Delta v-\left(A^{-1}(v)\right)_{t}=0
$$

According to Fichera-Oleìnik theory, we know that we can impose the Dirichlet homogeneous boundary condition (1.6). For the boundary layer equation (1.4), if the domain $\Omega=\{0<\tau<T, 0<\xi<X, 0<\eta<1\}$, then comparing equation (1.4) with equation (A.1), according to Fichera-Oleinnik theory, the initial and the boundary conditions for $w$ have the form

$$
\left.w\right|_{\tau=0}=w_{0}(\xi, \eta),\left.\quad w\right|_{\eta=1}=0,\left.\quad\left(\nu w w_{\eta}-v_{0} w+c(\tau, \xi)\right)\right|_{\eta=0}=0,
$$

where $v$ is the viscous coefficient, $v_{0}$ and $c(\tau, \xi)$ are known functions, one may refer to [1] for the details.

But, if equation (1.1) is strongly degenerate, then the inverse matrix $\left(a_{i j}\right)^{-1}$ is not-existent, we cannot deal with it as (A.4). Rewrite equation (1.1) as

$$
\frac{\partial u}{\partial t}=a^{i j}(u, x, t) \frac{\partial^{2} u}{\partial x_{i} \partial x_{j}}+a_{u}^{i j}(u, x, t) \frac{\partial u}{\partial x_{i}} \frac{\partial u}{\partial x_{j}}+a_{x_{i}}^{i j} u_{x_{j}}+b_{i u}(u, x, t) \frac{\partial u}{\partial x_{i}}+\frac{\partial b_{i}(u, x, t)}{\partial x_{i}}
$$


the domain is a cylinder $\Omega \times(0, T)$. If we let $t=x_{N+1}$ and regard the degenerate parabolic equation (A.6) as the form of a 'linear' degenerate elliptic equation as (A.1),

$$
\left(\widetilde{a}^{r s}\right)_{(N+1) \times(N+1)}=\left(\begin{array}{cc}
a^{i j} & 0 \\
0 & 0
\end{array}\right) .
$$

From this observation, according to Fichera-Oleǐnik theory, the initial value condition (1.5) is always necessary, but on the lateral boundary $\partial \Omega \times(0, T)$, by $a^{i j}(0, x, t)=0$, equation (A.6) is not only strongly degenerate in the interior of $\Omega$, but also on the boundary $\partial \Omega$. Then $\Sigma_{3}$ is an empty set. While

$$
\widetilde{b}_{s}(x, t)= \begin{cases}b_{i u}(u, x, t)+a_{u}^{i j}(u, x, t) \frac{\partial u}{\partial x_{j}}+a_{x_{j}}^{i j}(u, x, t), & 1 \leq s=i \leq N \\ -1, & s=N+1 .\end{cases}
$$

The portion of the boundary on which we can give the boundary value is

$$
\begin{aligned}
\Sigma_{p}= & \left\{x \in \partial \Omega:\left(b_{i u}(0, x, t)+a_{u}^{i j}(0, x, t) \frac{\partial u}{\partial x_{j}}+a_{x_{j}}^{i j}(0, x, t)\right.\right. \\
& \left.\left.-a_{x_{j}}^{i j}(0, x, t)-a_{u}^{i j}(0, x, t) \frac{\partial u}{\partial x_{j}}\right) n_{i}<0\right\} \\
= & \left\{x \in \partial \Omega: b_{i u}(0, x, t) n_{i}(x)<0\right\},
\end{aligned}
$$

where $\left\{n_{i}\right\}$ is the unit inner normal vector of $\partial \Omega$.

However, due to the strongly degenerate property of $\left(a^{i j}(u, x, t)\right)$, equation (A.6) generally only has a weak solution $u$, for example in our paper, $u \in \mathrm{BV}$, we cannot define the trace of $\frac{\partial u}{\partial x_{i}}$ on $\partial \Omega$. Fortunately, only if $b_{i}(s, x, t)$ is derivable, then

$$
\Sigma_{p}=\left\{x \in \partial \Omega: b_{i u}(0, x, t) n_{i}(x)<0\right\}
$$

has a definite sense. In the following, we will show that $\Sigma_{p}$ of (A.8) is in accordance with (1.13) in a special weak sense.

Recalling that, for any $\eta>0, \forall k \in R, \vec{n}=\left\{n_{i}\right\}$ is the inner unit normal vector of $\Sigma=\partial \Omega$, and for any given $t \in(0, T)$,

$$
\begin{aligned}
& \Sigma_{1 \eta k}=\left\{x \in \partial \Omega, S_{\eta}(k)\left[b_{i}(0, x, t)-b_{i}(k, x, t)\right] n_{i}(x)>0\right\}, \\
& \Sigma_{2 \eta k}=\left\{x \in \partial \Omega, S_{\eta}(k)\left[b_{i}(0, x, t)-b_{i}(k, x, t)\right] n_{i}(x) \leq 0\right\} .
\end{aligned}
$$

Let

$$
\Sigma_{1}=\bigcup_{\forall \eta>0, \forall k \in \mathbb{R}} \Sigma_{1 \eta k}, \quad \Sigma_{2}=\Sigma \backslash \Sigma_{1}
$$

We know that the boundary condition of equation (1.1) used in our paper is

$$
\left.\gamma u\right|_{\Sigma_{1} \times(0, T)}=0 .
$$


In fact, by the definition of $\Sigma_{1 \eta k}$, we know that

$$
0<S_{\eta}(k)\left[b_{i}(0, x, t)-b_{i}(k, x, t)\right] n_{i}(x)=-k S_{\eta}(k) b_{i}^{\prime}(\zeta, x, t) n_{i}(x)
$$

where $\zeta \in(k, 0), b_{i}^{\prime}(\zeta, x, t)=\left.b_{i u}(u, x, t)\right|_{u=\zeta}$. If we let $\eta \rightarrow 0$, then

$$
b_{i}^{\prime}(\zeta, x, t) n_{i}(x)<0
$$

Let $k \rightarrow 0$. We know that

$$
b_{i}^{\prime}(0, x, t) n_{i}(x)<0
$$

which is in accordance with (A.8).

\section{Appendix 2: The comments on Definition 1.1}

To explain the reasonableness of Definition 1.1, suppose that equation (1.1) has a classical solution $u$. For any $\varphi_{1} \in C^{2}\left(\overline{Q_{T}}\right), \varphi_{1} \geq 0,\left.\nabla \varphi_{1}\right|_{\Sigma}=0$, supp $\varphi_{1} \subset \bar{\Omega} \times(0, T), k \in \mathbb{R}, \eta>0$. Multiplying (1.1) by $\varphi_{1} S_{\eta}(u-k)$ and integrating over $Q_{T}$, we have

$$
\begin{aligned}
& \iint_{Q_{T}} I_{\eta}(u-k) \varphi_{1 t} d x d t+\iint_{Q_{T}} A_{\eta}^{i j}(u, x, t, k) \varphi_{1 x_{i} x_{j}} d x d t-\iint_{Q_{T}} B_{\eta}^{i}(u, x, t, k) \varphi_{1 x_{i}} d x d t \\
& \quad-\iint_{Q_{T}} a^{i j}(u, x, t) u_{x_{i}} u_{x_{j}} S_{\eta}^{\prime}(u-k) \varphi_{1} d x d t+\iint_{Q_{T}} \int_{k}^{u} a_{x_{j}}^{i j}(s, x, t) S_{\eta}(s-k) d s \varphi_{1 x_{i}} d x d t \\
& \quad+S_{\eta}(k) \int_{0}^{T} \int_{\Sigma} a^{i j}(u, x, t) \frac{\partial u}{\partial x_{j}} n_{i} \varphi_{1} d t d \sigma+S_{\eta}(k) \int_{0}^{T} \int_{\Sigma} A_{\eta}^{i j}(0, x, t, k) \varphi_{1 x_{i}} n_{j} d t d \sigma \\
& \quad+S_{\eta}(k) \int_{0}^{T} \int_{\Sigma_{1 \eta k}}\left[b_{i}(0, x, t)-b_{i}(k, x, t)\right] n_{i} \varphi_{1} d t d \sigma \\
& \quad+S_{\eta}(k) \int_{0}^{T} \int_{\Sigma_{2 \eta k}}\left[b_{i}(0, x, t)-b_{i}(k, x, t)\right] n_{i} \varphi_{1} d t d \sigma=0
\end{aligned}
$$

Taking $\varphi_{2} \in C^{2}\left(\bar{Q}_{T}\right),\left.\varphi_{1}\right|_{\partial \Omega \times[0, T]}=\left.\varphi_{2}\right|_{\partial \Omega \times[0, T]}, \operatorname{supp} \varphi_{2} \subset \bar{\Omega} \times(0, T)$,

$$
\begin{aligned}
& S_{\eta}(k) \int_{0}^{T} \int_{\Sigma} a^{i j}(u, x, t) \frac{\partial u}{\partial x_{j}} n_{i} \varphi_{1} d t d \sigma \\
& =S_{\eta}(k)\left\{-\iint_{Q_{T}} a^{i j}(u, x, t) \frac{\partial u}{\partial x_{j}} \varphi_{2 x_{i}} d x d t+\iint_{Q_{T}} \frac{\partial b_{i}(0, x, t)}{\partial x_{i}} \varphi_{2} d x d t\right. \\
& \quad-\iint_{Q_{T}}\left[b_{i}(u, x, t)-b_{i}(0, x, t)\right] \frac{\partial \varphi_{2}}{\partial x_{i}} d x d t+\iint_{Q_{T}} u \frac{\partial \varphi_{2}}{\partial t} d x d t \\
& \left.\quad-\int_{0}^{T} \int_{\Sigma}\left[b_{i}(0, x, t)-b_{i}(0, x, t)\right] n_{i} \varphi_{2} d t d \sigma\right\} \\
& \iint_{Q_{T}} a^{i j}(u, x, t) \frac{\partial u}{\partial x_{j}} \varphi_{2 x_{i}} d x d t \\
& =-\int_{0}^{T} \int_{\Sigma} A^{i j}(0, x, t) \varphi_{2 x_{i}} n_{j} d t d \sigma
\end{aligned}
$$




$$
\begin{aligned}
& -\iint_{Q_{T}} A^{i j}(u, x, t) \varphi_{2 x_{i} x_{j}} d x d t-\iint_{Q_{T}} \int_{0}^{u} a_{x_{j}}^{i j}(s, x, t) d s \varphi_{2 x_{i}} d x d t \\
= & -\iint_{Q_{T}} A^{i j}(u, x, t) \varphi_{2 x_{i} x_{j}} d x d t-\iint_{Q_{T}} \int_{0}^{u} a_{x_{j}}^{i j}(s, x, t) d s \varphi_{2 x_{i}} d x d t .
\end{aligned}
$$

For $\left.\nabla \varphi_{1}\right|_{\Sigma}=0$, and by $A^{i j}(0, x, t)=0$, from (B.1)-(B.3), we have

$$
\begin{aligned}
& \iint_{Q_{T}} I_{\eta}(u-k) \varphi_{1 t} d x d t+\iint_{Q_{T}} A_{\eta}^{i j}(u, x, t, k) \varphi_{1 x_{i} x_{j}} d x d t-\iint_{Q_{T}} B_{\eta}^{i}(u, x, t, k) \varphi_{1 x_{i}} d x d t \\
& \quad+\iint_{Q_{T}} \int_{0}^{u} a_{x_{j}}^{i j}(s, x, t) S_{\eta}(s-k) d s \varphi_{1 x_{i}} d x d t \\
& \quad+S_{\eta}(k)\left[\iint_{Q_{T}} A^{i j}(u, x, t) \varphi_{2 x_{i} x_{j}} d x d t+\iint_{Q_{T}} \frac{\partial b_{i}(0, x, t)}{\partial x_{i}} \varphi_{2} d x d t\right. \\
& \left.\quad-\iint_{Q_{T}}\left(b_{i}(u, x, t)-b_{i}(0, x, t)\right) \frac{\partial \varphi_{2}}{\partial x_{i}} d x d t+\iint_{Q_{T}} u \frac{\partial \varphi_{2}}{\partial t} d x d t\right] \\
& \quad+S_{\eta}(k) \iint_{Q_{T}} \int_{0}^{u} a_{x_{j}}^{i j}(s, x, t) d s \varphi_{2 x_{i}} d x d t-\iint_{Q_{T}} a^{i j}(u, x, t) u_{x_{i}} u_{x_{j}} S_{\eta}^{\prime}(u-k) \varphi_{1} d x d t \\
& \quad+S_{\eta}(k) \int_{0}^{T} \int_{\Sigma_{1 \eta k}}\left[b_{i}(0, x, t)-b_{i}(k, x, t)\right] n_{i} \varphi_{1} d t d \sigma \\
& =-S_{\eta}(k) \int_{0}^{T} \int_{\Sigma_{2 \eta k}}\left[b_{i}(0, x, t)-b_{i}(k, x, t)\right] n_{i} \varphi_{1} d t d \sigma .
\end{aligned}
$$

Now, we give some comments.

(i) First, the classical solution $u$ induces an integral equality (B.4), while the weak solution formula defined by (1.16) can be rewritten as

$$
\begin{aligned}
\iint_{Q_{T}} & {\left[I_{\eta}(u-k) \varphi_{1 t}-B_{\eta}^{i}(u, x, t, k) \varphi_{1 x_{i}}+A_{\eta}^{i j}(u, x, t, k) \frac{\partial^{2} \varphi_{1}}{\partial x_{i} \partial x_{j}}\right.} \\
& \left.-S_{\eta}^{\prime}(u-k) \sum_{j=1}^{N}\left|g^{j}\right|^{2} \varphi_{1}\right] d x d t+\iint_{Q_{T}} \int_{k}^{u} a_{x_{j}}^{i j}(s, x, t) S_{\eta}(s-k) d s \varphi_{1 x_{i}} d x d t \\
& +S_{\eta}(k) \iint_{Q_{T}}\left[u \varphi_{2 t}-\left(b_{i}(u, x, t)-b_{i}(0, x, t)\right) \varphi_{2 x_{i}}+A^{i j}(u, x, t) \frac{\partial^{2} \varphi_{2}}{\partial x_{i} \partial x_{j}}\right. \\
& \left.+\frac{\partial b_{i}(0, x, t)}{\partial x_{i}} \varphi_{2}\right] d x d t+S_{\eta}(k) \int_{0}^{T} \int_{\Sigma_{1 \eta k}}\left[b_{i}(0, x, t)-b_{i}(k, x, t)\right] n_{i} \varphi_{1} d t d \sigma \\
\geq & -S_{\eta}(k) \int_{0}^{T} \int_{\Sigma_{2 \eta k}}\left(b_{i}(0, x, t)-b_{i}(k, x, t)\right) n_{i} \varphi_{1} d t d \sigma
\end{aligned}
$$

which is just an inequality, this is due to the following weak convergence property: assuming that $U \subset \mathbb{R}^{N}$ is an open bounded set and as $k \rightarrow \infty, f_{k} \rightarrow f$ weakly in $L^{q}(U), 1 \leq q<\infty$, then

$$
\lim _{k \rightarrow \infty} \inf \left\|f_{k}\right\|_{L^{q(U)}}^{q} \geq\|f\|_{L^{q}(U)}^{q}
$$

which has been quoted before as Lemma 2.7 . 
Generally, inequality (B.6) cannot be an equality. This is why we can only define the weak solution as (1.16) (i.e. (B.5)) instead of (B.4).

(ii) Second, Definition 1.1 is equivalent to the following.

Definition B.1 A function $u$ is said to be the entropy solution of equation (1.1)-(1.5)-(1.13), if:

1. $u \in \operatorname{BV}\left(Q_{T}\right) \cap L^{\infty}\left(Q_{T}\right)$, and there exist functions $g^{i} \in L^{2}\left(Q_{T}\right), i=1,2, \ldots, N$, such that

$$
\iint_{Q_{T}} g^{i}(x, t) \varphi(x, t) d x d t=\iint_{Q_{T}} \widehat{\gamma}^{i j}(u, x, t) \varphi(x, t) \frac{\partial u}{\partial x_{j}} d x d t
$$

where $\varphi(x, t) \in L^{2}\left(Q_{T}\right),\left(\gamma^{i j}\right)$ is the square root of $\left(a^{i j}\right)$, and

$$
\widehat{\gamma}^{i j}(u, x, t)=\int_{0}^{1} \gamma^{i j}\left(s u^{+}+(1-s) u^{-}, x, t\right) d s .
$$

2. For any $\varphi \in C_{0}^{2}\left(Q_{T}\right)$, for any $k \in \mathbb{R}$, for any small $\eta>0$, $u$ satisfies

$$
\begin{aligned}
& \iint_{Q_{T}}\left[I_{\eta}(u-k) \varphi_{t}-B_{\eta}^{i}(u, x, t, k) \varphi_{x_{i}}+A_{\eta}^{i j}(u, x, t, k) \frac{\partial^{2} \varphi}{\partial x_{i} \partial x_{j}}\right. \\
& \left.-S_{\eta}^{\prime}(u-k) \sum_{j=1}^{N}\left|g^{j}\right|^{2} \varphi\right] d x d t \\
& \quad+\iint_{Q_{T}} \int_{k}^{u} a_{x_{j}}^{i j}(s, x, t) S_{\eta}(s-k) d s \varphi_{x_{i}} d x d t \geq 0 .
\end{aligned}
$$

3. The boundary value is satisfied in the sense of the trace,

$$
\left.\gamma u\right|_{\Sigma_{1 \eta k} \times(0, T)}=0 .
$$

4. The initial value is satisfied in the sense of the following equality:

$$
\lim _{t \rightarrow 0} \int_{\Omega}\left|u(x, t)-u_{0}(x)\right| d x=0
$$

Comparing with Definition 1.1, Definition B.1 seems simpler; the reason we choose to adopt Definition 1.1 is that the inequality (2.3) clearly shows the partial-boundary condition, and the definition can be used to deal with the corresponding problem if we have equation (1.1) with no homogeneous boundary value condition.

(iii) By (B.7), we have

$$
\begin{aligned}
& \iint_{Q_{T}}\left[I_{\eta}(u-k) \varphi_{t}-B_{\eta}^{i}(u, x, t, k) \varphi_{x_{i}}+A_{\eta}^{i j}(u, x, t, k)\right] \frac{\partial^{2} \varphi}{\partial x_{i} \partial x_{j}} d x d t \\
& \quad+\iint_{Q_{T}} \int_{k}^{u} a_{x_{j}}^{i j}(s, x, t) S_{\eta}(s-k) d s \varphi_{x_{i}} d x d t \geq 0 .
\end{aligned}
$$


Let $\eta \rightarrow 0$ in this inequality. We have

$$
\begin{aligned}
& \iint_{Q_{T}}\left\{|u-k| \varphi_{t}-\operatorname{sgn}(u-k)\left[b_{i}(u)-b_{i}(k)\right]+\left|A^{i j}(u, x, t)-A^{i j}(k, x, t)\right| \frac{\partial^{2} \varphi}{\partial x_{i} \partial x_{j}}\right\} d x d t \\
& \quad+\iint_{Q_{T}} \int_{k}^{u} a_{x_{j}}^{i j}(s, x, t) \operatorname{sgn}(s-k) d s \varphi_{x_{i}} d x d t \geq 0
\end{aligned}
$$

Inequality (B.9) is the entropy solution defined in [15-17]. In other words, there was a time that the term $-S_{\eta}^{\prime}(u-k) \sum_{j=1}^{N}\left|g^{j}\right|^{2} \varphi d x d t$ was regarded as 'redundant' and was drawn away. Actually, we have seen that the term implies very important information on the uniqueness.

(iv) If considering the equation

$$
\frac{\partial u}{\partial t}=\frac{\partial^{2} A(u)}{\partial x^{2}}+\frac{\partial B(u)}{\partial x}, \quad(x, t) \in \mathbb{R} \times(0, T),
$$

Vol'pert and Hudjaev in [39] defined $u \in \mathrm{BV}\left(Q_{T}\right) \cap L^{\infty}\left(Q_{T}\right)$ is said to be a weak solution of (B.10), if $\frac{\partial A(u)}{\partial x} \in L_{l o c}^{1}\left(Q_{T}\right)$, and for any $0 \leq \varphi \in C_{0}^{\infty}\left(Q_{T}\right)$, any $k \in \mathbb{R}$,

$$
\begin{aligned}
& \iint_{Q_{T}} \operatorname{sgn}(u-k)\left[(u-k) \frac{\varphi}{\partial t}-\frac{\partial A(u)}{\partial x} \frac{\partial \varphi}{\partial x}\right] d x d t \\
& \quad-\iint_{Q_{T}} \operatorname{sgn}(u-k)\left[(B(u)-B(k)) \frac{\partial \varphi}{\partial x}\right] d x d t \geq 0 .
\end{aligned}
$$

We know that only under the condition $\frac{\partial A(u)}{\partial x} \in L^{\infty}\left(Q_{T}\right) \cap \mathrm{BV}_{x}\left(Q_{T}\right)$ the uniqueness of the solutions in the sense of (B.11) is true.

However, in the present case of strong degeneration, since for the limit function $u$ of certain subsequence of $\left.\left\{u_{\varepsilon}\right\}, a^{\widehat{i j}(u, x, t}\right) \frac{\partial u}{\partial x_{j}}$ cannot be defined by the trace $\left.\gamma\left(a^{\hat{i j}(u, x, t}\right) \frac{\partial u}{\partial x_{j}}\right)$ on $\Sigma$, we have to make a detour to avoid $\left.\gamma\left(a^{\widehat{i j}(u, x, t}\right) \frac{\partial u}{\partial x_{j}}\right)$ in defining the entropy solution. So, an essential improvement of our paper (also [5-13]) is to get the uniqueness of the solutions without any bounded restrictions in $\frac{\partial A(u)}{\partial x}$.

\section{Competing interests}

The author declares to have no competing interests.

\section{Acknowledgements}

The paper is supported by NSF of China (no. 11371297) and by NSF of Fujian Province (no. 2015J01592), China.

Received: 13 May 2015 Accepted: 30 December 2015 Published online: 16 January 2016

\section{References}

1. Oleinik, OA, Samokhin, VN: Mathematical Models in Boundary Layer Theorem. Chapman \& Hall/CRC, Boca Raton (1999)

2. Wu, Z, Zhao, J, Yin, J, Li, H: Nonlinear Diffusion Equations. World Scientific, Singapore (2001)

3. $\mathrm{Wu}, \mathrm{Z}, \mathrm{Zhao}, \mathrm{J}$ : The first boundary value problem for quasilinear degenerate parabolic equations of second order in several variables. Chin. Ann. Math., Ser. B 4, 57-76 (1983)

4. $\mathrm{Wu}, \mathrm{Z}, \mathrm{Zhao}, \mathrm{J}$ : Some general results on the first boundary value problem for quasilinear degenerate parabolic equations, 2. Chin. Ann. Math., Ser. B 4, 319-328 (1983)

5. Yin, J, Wang, C: Evolutionary weighted p-Laplacian with boundary degeneracy. J. Differ. Equ. 237, 421-445 (2007)

6. Kobayasi, K, Ohwa, H: Uniqueness and existence for anisotropic degenerate parabolic equations with boundary conditions on a bounded rectangle. J. Differ. Equ. 252, 137-167 (2012)

7. Li, Y, Wang, Q: Homogeneous Dirichlet problems for quasilinear anisotropic degenerate parabolic-hyperbolic equations. J. Differ. Equ. 252, 4719-4741 (2012)

8. Lions, PL, Perthame, B, Tadmor, E: A kinetic formation of multidimensional conservation laws and related equations. J. Am. Math. Soc. 7, 169-191 (1994) 
9. Escobedo, M, Vazquez, JL, Zuazua, E: Entropy solutions for diffusion-convection equations with partial diffusivity. Trans. Am. Math. Soc. 343, 829-842 (1994)

10. Guarguaglini, FR, Milišić, V, Terracina, A: A discrete BGK approximation for strongly degenerate parabolic problems with boundary conditions. J. Differ. Equ. 202, 183-207 (2004)

11. Málek, J, Necas, J, Rokyta, M, Ruzicka, M: Weak and Measure-Valued Solutions to Evolutionary PDES. Applied Mathematics and Mathematical Computation, vol. 13. Chapman \& Hall, London (1996)

12. Mascia, C, Porretta, A, Terracina, A: Nonhomogeneous Dirichlet problems for degenerate parabolic-hyperbolic equations. Arch. Ration. Mech. Anal. 163(2), 87-124 (2002)

13. Vallet, G: Dirichlet problem for a degenerated hyperbolic-parabolic equation. Adv. Math. Sci. Appl. 15, 423-450 (2005)

14. Zhan, H: The solution of a hyperbolic-parabolic mixed-type equation on half-space domain. J. Differ. Equ. 259, 1449-1481 (2015)

15. Vol'pert, Al, Hudjaev, SI: On the problem for quasilinear degenerate parabolic equations of second order. Mat. Sb. 3, 374-396 (1967) (in Russian)

16. Zhao, J: Uniqueness of solutions of quasilinear degenerate parabolic equations. Northeast. Math. J. 1(2), 153-165 (1985)

17. Vol'pert, Al: BV space and quasilinear equations. Mat. Sb. 73, 255-302 (1967)

18. Zhan, H: Homogeneous Dirichlet condition of a anisotropic degenerate parabolic equation. Bound. Value Probl. 2015, 22 (2015)

19. Enrico, G: Minimal Surfaces and Functions of Bounded Variation. Birkhäuser, Basel (1984)

20. Evans, LC: Weak convergence methods for nonlinear partial differential equations. In: Conference Board of the Mathematical Sciences. Regional Conferences Series in Mathematics, vol. 74 (1998)

21. Gu, L: Second Order Parabolic Partial Differential Equations. The Publishing Company of Xiamen University, China (2004)

22. Zhao, J, Zhan, H: Uniqueness and stability of solution for Cauchy problem of degenerate quasilinear parabolic equations. Sci. China Ser. A 48, 583-593 (2005)

23. Ragusa, MA: The Cauchy-Dirichlet problem for parabolic equations with VMO coefficients. Math. Comput. Model. 42(11), 1245-1254 (2005)

24. Ragusa, MA: Cauchy-Dirichlet problem associated to divergence form parabolic equations. Commun. Contemp. Math. 6(3), 377-393 (2004)

25. Bardos, C, LeRoux, AY, Nédélec, JC: First order quasilinear equations with boundary condition. Commun. Partial Differ. Equ. 4, 1017-1024 (1979)

26. Otto, F: Initial-boundary value problem for a scalar conservation laws. C. R. Math. Acad. Sci. Paris, Sér. I 322, 729-734 (1996)

27. Carrillo, J: Entropy solutions for nonlinear degenerate problems. Arch. Ration. Mech. Anal. 147, 269-361 (1999)

28. Michel, A, Vovelle, J: Entropy formulation for parabolic degenerate equations with general Dirichlet boundary conditions and application to the convergence of FV methods. SIAM J. Numer. Anal. 41, 2262-2293 (2003)

29. Kobayasi, K: A kinetic approach to comparison properties for degenerate parabolic-hyperbolic equations with boundary conditions. J. Differ. Equ. 230, 682-701 (2006)

30. Chen, GQ, Perthame, B: Well-posedness for non-isotropic degenerate parabolic-hyperbolic equations. Ann. Inst. Henri Poincaré, Anal. Non Linéaire 20(4), 645-668 (2003)

31. Bendahamane, M, Karlsen, KH: Reharmonized entropy solutions for quasilinear anisotropic degenerate parabolic equations. SIAM J. Math. Anal. 36(2), 405-422 (2004)

32. Bendahmane, M, Karlsen, KH: Uniqueness of entropy solutions for quasilinear anisotropic degenerate parabolic equation. Contemp. Math. 371, 1-27 (2005)

33. Andreianov, BP, Bendahmane, M, Karlsen, KH: Discrete duality finite volume schemes for doubly nonlinear degenerate hyperbolic-parabolic equations. Preprint. http://www.math.ntnu.no/conservation/2009/001.html (2009)

34. Andreianov, BP, Bendahmane, $\mathrm{M}$, Karlsen, $\mathrm{KH}$, Ouaro, S: Well-posedness results for triply nonlinear degenerate parabolic equations. J. Differ. Equ. 247, 277-302 (2009)

35. Fichera, G: Sulle equazioni differenziatli lineari ellittico-paraboliche del secondo ordine. Atti Accad. Naz. Lincei Mem. Ci. Sci. Fis. Mat. Natur. Sez. I 5(8), 1-30 (1956)

36. Fichera, G: On a unified theory of boundary value problems for elliptic-parabolic equations of second order. In: Boundary Problems, Differential Equations, pp. 97-120. University of Wisconsin Press, Madison (1960)

37. Oleǐnik, OA: A problem of Fichera. Dokl. Akad. Nauk SSSR 154, 1297-1300 (1964); English transl.: Sov. Math. Dokl. 5 1129-1133 (1964)

38. Oleŭnik, OA: Linear equations of second order with nonnegative characteristic form. Mat. Sb. 69, 111-140 (1966); English transl.: Transl. Am. Math. Soc. 65(2), 167-199 (1967)

39. Volpert, Al, Hudjaev, SI: Cauchy's problem for degenerate second order quasilinear parabolic equation. Math. USSR Sb. 7, 365-387 (1969) 